\title{
Threshold Dynamics and Competitive Exclusion in a Virus Infection Model with General Incidence Function and Density-Dependent Diffusion
}

\author{
Xiaosong Tang $\mathbb{D},{ }^{1}$ Zhiwei Wang, ${ }^{1}$ and Jianping Yang ${ }^{2}$ \\ ${ }^{1}$ School of Mathematics and Physics, Jinggangshan University, Ji'an 343009, China \\ ${ }^{2}$ School of Electronics and Information Engineering, Jinggangshan University, Ji'an 343009, China \\ Correspondence should be addressed to Xiaosong Tang; tangxs40@sina.com
}

Received 27 August 2019; Revised 8 December 2019; Accepted 19 December 2019; Published 9 April 2020

Academic Editor: Mahdi Jalili

Copyright ( 12020 Xiaosong Tang et al. This is an open access article distributed under the Creative Commons Attribution License, which permits unrestricted use, distribution, and reproduction in any medium, provided the original work is properly cited.

In this paper, we investigate single-strain and multistrain viral infection models with general incidence function and densitydependent diffusion subject to the homogeneous Neumann boundary conditions. For the single-strain viral infection model, by using the linearization method and constructing appropriate Lyapunov functionals, we obtain that the global threshold dynamics of the model is determined by the reproductive numbers for viral infection $\mathscr{R}_{0}$. For the multistrain viral infection model, we have discussed the competitive exclusion problem. If the reproduction number $\mathscr{R}_{i}$ for strain $i$ is maximal and larger than one, the steady state $E_{i}$ corresponding to the strain $i$ is globally stable. Thus, competitive exclusion happens and all other strains die out except strain $i$. Meanwhile, we can prove that the single-strain and multistrain viral infection models are well posed. Furthermore, numerical simulations are also carried out to illustrate the theoretical results, which is seldom seen in the relevant known literatures.

\section{Introduction}

Nowadays, more and more people in the world are dying of various diseases such as AIDS, avian influenza, cholera, Ebola, and Zika virus. To explore the mechanisms of these diseases, scientists have proposed plenty of mathematical models describing the transmission of disease such as the infectious disease model (SI, SIR, SEI, and SIS) and the within-host virus model (HBV, HCV, HIV, Ebola, and Zika). Since Nowak et al. $[1,2]$ has established the basic within-host virus dynamics model, more and more scholars have devoted to investigate the within-host virus dynamics model and obtained so many significant dynamical results [3-16].

Assuming that the motion of the virus belongs to the Fickian diffusion [17], Wang et al. [5] have firstly investigated a delayed diffusive hepatitis B virus model. Here, we have to mention that the equation with Fickian diffusion implies that random walkers in the system have no interactions. In fact, random walkers in the system may have interactions and the density-dependent diffusion happens [17] and we may establish the equation with density-dependent diffusion. So, parabolic or elliptic systems with density-dependent diffusion have been widely studied by many researchers [18-21]. More recently, Wang et al. have investigated a virus infection model with density-dependent diffusion and Holling II type [6] or Beddington-DeAngelis type incidence function [7] subjected to the homogeneous Neumann boundary conditions. And, they have obtained the significant results of threshold dynamics and competitive exclusion.

It is well known that there are many factors which affect the dynamics of within-host models. One crucial component is incidence function, which can be classified into many different types, such as bilinear type [2], Holling II type [22], Beddington-DeAngelis type [23], and Crowley-Martin type 
[24]. Many researchers have concentrated on these withinhost models or epidemic models and have obtained some interesting results such as global threshold dynamics $[8,9]$, delay-induced Hopf bifurcation [10, 11], competitive exclusion $[6,7]$, traveling wave $[12,13]$, and pattern formation [14-16]. Recently, Korobeinikov [25] studied an ordinary differential equation model with general incidence function and dose-dependent parasite reproduction and virulence. Huang et al. [26] investigated a viral infection model involving time delay and general incidence function. McCluskey and Yang [27] considered a diffusive viral infection model with general incidence function and time delay. Hattaf and Yousfi [28] studied a diffusive HBV model with two delays and general incidence function. Miao et al. [29] considered the global stability of a diffusive humoral immunity virus infection model with time delay and general incidence function and so on.

Based on the aforementioned works, we firstly propose a single-strain viral infection model with density-dependent diffusion and general incidence function as follows:

$$
\left\{\begin{array}{l}
\frac{\partial u(x, t)}{\partial t}=\nabla \cdot\left(D_{u}(u) \nabla u\right)+\lambda-d u(x, t)-g u((x, t), v(x, t)) \\
\frac{\partial w(x, t)}{\partial t}=\nabla \cdot\left(D_{w}(w) \nabla w\right)+g(u(x, t), v(x, t))-\delta w(x, t), \\
\frac{\partial v(x, t)}{\partial t}=\nabla \cdot\left(D_{v}(v) \nabla v\right)+p w(x, t)-c v(x, t)
\end{array}\right.
$$

where $D_{u}(u)=u^{m_{1}}, D_{w}(w)=w^{m_{2}}$, and $D_{v}(v)=v^{m_{3}}$ are the density-dependent diffusion coefficients for $m_{i}>0$, $i=1,2,3 ; u(x, t)$ represents the densities of uninfected cells; $w(x, t)$ represents the densities of infected cells; $v(x, t)$ represents the densities of free virus at position $x$ and at time $t$, respectively; constant $\lambda$ represents the production rate of the uninfected cells; the death rates of uninfected cells, infected cells, and free virus are represented by the parameters $d, \delta$, and $c$, respectively; the infected cells produce the free virions at a rate $p w$; further, we assume that the incidence function in this paper is a general incidence function given by $g(u, v)$, which represents the contacts between target cells and viruses; and the general incidence function $g(u, v)$ satisfies the following conditions [27].

$\left(H_{1}\right) g: \mathbb{R}_{+}^{2} \longrightarrow \mathbb{R}_{+}$is differentiable; there exists $\eta>0$ such that $g(u, v) \leq \eta u v$ for all $u, v \geq 0 ; g(u, v)>0$ for all $u, v>0 ; g(u, 0)=g(0, v)=0$ for all $u, v \geq 0 ;(\partial g(u, v) / \partial u)>$ 0 for all $u \geq 0$ and $v>0$; $(\partial g(u, v) / \partial v) \geq 0$ for all $u, v \geq 0$; $v(\partial g(u, v) / \partial v) g(u, v) \leq 0$ for all $u, v \geq 0$.

These assumptions are biologically motivated, see [27] in detail. Moreover, for $a, b, c, \beta>0$, we choose the incidence functions such as $g(u, v)=(\beta u v /(1+b v))$ [22], $g(u, v)=$ $(\beta u v /(1+a u+b v))[23]$, and $g(u, v)=(\beta u v /(1+a u+b v+$ cuv)) [24], which satisfy $\left(H_{1}\right)$ clearly.

Model (1) we considered in this paper satisfies the following Neumann boundary conditions:

$$
\begin{aligned}
& D_{u}(u) \frac{\partial u}{\partial \nu}=0, \\
& D_{w}(w) \frac{\partial w}{\partial \nu}=0, \\
& D_{v}(v) \frac{\partial v}{\partial \nu}=0, \\
& \text { on } \partial \Omega \times(0,+\infty),
\end{aligned}
$$

and initial conditions

$$
\begin{gathered}
u(x, 0)=\phi_{1}(x) \geq 0, \\
w(x, 0)=\phi_{2}(x) \geq 0, \\
v(x, 0)=\phi_{3}(x) \geq 0, \\
x \in \bar{\Omega} .
\end{gathered}
$$

Here, we give out a bounded domain $\Omega$ in $\mathbb{R}^{n}$ and its boundary $\partial \Omega$ is smooth, and the outward normal derivative on $\partial \Omega$ is denoted by $(\partial / \partial \nu)$. The nonnegative functions $\phi_{i}(x, 0) \quad(i=1,2,3)$ are Hölder continuous and satisfy $\left(\partial \phi_{i} / \partial \nu\right)=0(i=1,2,3)$ on $\partial \Omega$.

For (1), we can obtain its steady-state system,

$$
\left\{\begin{array}{l}
-\nabla \cdot\left(D_{u}(u) \nabla u\right)=\lambda-d u(x, t)-g(u(x, t), v(x, t))=f_{1}(u, v, w), \\
-\nabla \cdot\left(D_{w}(w) \nabla w\right)=g(u(x, t), v(x, t))-\delta w(x, t)=f_{2}(u, v, w), \\
-\nabla \cdot\left(D_{v}(v) \nabla v\right)=p w(x, t)-c v(x, t)=f_{3}(u, v, w)
\end{array}\right.
$$

which satisfies boundary conditions (2). We denote

$$
\mathbf{f}(u, v, w)=\left(f_{1}(u, v, w), f_{2}(u, v, w), f_{3}(u, v, w)\right),
$$

and let $f_{i}(u, v, w)(i=1,2,3)$ satisfy the following conditions.

$\left(H_{2}\right)$ The following relations

$$
\begin{aligned}
& \gamma_{1} u^{m_{1}}+\frac{\partial f_{1}}{\partial u} \geq 0, \\
& \gamma_{2} w^{m_{2}}+\frac{\partial f_{2}}{\partial w} \geq 0, \\
& \gamma_{3} v^{m_{3}}+\frac{\partial f_{3}}{\partial v} \geq 0,
\end{aligned}
$$


hold for constants $\gamma_{i} \geq 0 \quad(i=1,2,3)$.

Since Gause has discussed the principles that govern species competitive exclusion [30], competitive exclusion has been considered in such settings such as within-host models, ecological models, and epidemiological models. Here, we have to point out that Bremermann and Thieme [31] have given out the first rigorous proof of the competitive exclusion principle in the epidemiological model setting. For the multistrain SIR model, they have proved that the strain with the largest reproduction number persists in the population, while the remaining strains die out. This principle was also used in within-host models and has been studied by many scholars [32-37]. For instance, De Leenheer and Pilyugin [32] proved the global competitive exclusion in an ODE version with a multistrain virus. Feng and Velasco-
Hernández [33] discussed competitive exclusion in a vectorhost model for the dengue fever. Iggidr et al. [34] analyzed globally a new malaria within-host model through a competitive exclusion principle. Wang et al. investigated the competitive exclusion in the virus infection model with density-dependent diffusion and two types of incidence functions [6, 7]. Duan et al. [36] considered the competitive exclusion in the diffusive virus infection model with age of infection and general incidence function, and so on. So, in this paper, we further investigate the competitive exclusion problem for the multistrain virus infection model. Setting $i=1,2, \ldots, n$, we can obtain the following multistrain viral infection model with density-dependent diffusion and general incidence function:

$$
\left\{\begin{array}{l}
\frac{\partial u(x, t)}{\partial t}=\nabla \cdot\left(D_{u}(u) \nabla u\right)+\lambda-d u(x, t)-\sum_{i=1}^{n} g_{i}\left(u(x, t), v_{i}(x, t)\right), \\
\frac{\partial w_{i}(x, t)}{\partial t}=\nabla \cdot\left(D_{w_{i}}\left(w_{i}\right) \nabla w_{i}\right)+g_{i}\left(u(x, t), v_{i}(x, t)\right)-\delta_{i} w_{i}(x, t), \\
\frac{\partial v_{i}(x, t)}{\partial t}=\nabla \cdot\left(D_{v_{i}}\left(v_{i}\right) \nabla v_{i}\right)+p_{i} w_{i}(x, t)-c_{i} v_{i}(x, t), \quad i=1,2, \ldots, n .
\end{array}\right.
$$

Here, we have to stress that the Neumann boundary conditions, initial conditions, and steady-state system of Model (7) are described in Section 3. However, to the best of our knowledge, there are no results on the above models (1) and (7) such as global threshold dynamics and competitive exclusion. So, in this paper, we shall focus on the global threshold dynamics for the single-strain viral infection model (1) and competitive exclusion for the multi-strain viral infection model (7). It is worthwhile to mention that the incidence function discussed in $[6,7]$ is a special incidence function. But, the opposite case is more difficult and complicated. Through constructing an appropriate Lyapunov functional, Lemma 2, and mathematic analysis skills, we overcome the difficulties successfully and obtain the global threshold dynamics for the single-strain viral infection model (1) and competitive exclusion for the multi-strain viral infection model (7).

The rest of this paper is organized as follows: in Section 2 , for the single-strain viral infection model (1), we shall prove the existence, uniqueness, and boundedness of solutions to problems (1)-(4) and consider the global threshold dynamics of the model through constructing appropriate Lyapunov functionals, which is determined by the reproductive numbers for viral infection $\mathscr{R}_{0}$. In Section 3, for the multistrain viral infection model (7), we also prove the existence, uniqueness, and boundedness of solutions to problems (7) and (42) and discuss the competitive exclusion problem. If the reproduction number $\mathscr{R}_{i}$ for strain $i$ is maximal and larger than one, the steady state $E_{i}$ corresponding to the strain $i$ is globally stable. Thus, competitive exclusion happens and all other strains die out except strain $i$. In Section 4 , we illustrate our results with numerical simulations, which support and extend the theoretical results. The paper ends with a conclusion.

\section{Single-Strain Viral Infection Model}

In this section, we shall prove firstly that the single-strain viral infection model (1) is well posed by the method used in [18]. Then, we continue to discuss the existence of equilibria for model (1). Finally, through constructing appropriate Lyapunov functionals, we investigate the global threshold dynamics of model (1), which is determined by the reproductive numbers for viral infection $\mathscr{R}_{0}$.

2.1. Well Posedness. In this section, we give out some definitions and results first, which can help us to prove that the solutions to problems (1)-(4) exist and are unique and bounded. Similar to the definition method in $[6,7]$, we can definite that two pairs of functions $\widetilde{U}=(\widetilde{u}, \widetilde{w}, \widetilde{v})$, $\widehat{U}=(\widehat{u}, \widehat{w}, \widehat{v}) \quad$ in $C(\bar{\Omega} \times[0, T]) \cap C^{1,2}(\Omega \times[0, T])$ and $\widetilde{U}_{s}=\left(\widetilde{u}_{s}, \widetilde{w}_{s}, \widetilde{v}_{s}\right), \quad \widehat{U}_{s}=\left(\widehat{u}_{s}, \widehat{w}_{s}, \widehat{v}_{s}\right) \quad$ in $C(\bar{\Omega} \times[0, T]) \cap$ $C^{1,2}(\Omega \times[0, T])$ are called coupled upper and lower solutions to system (1)-(3) and to system (4), respectively. Then, we can easily conclude that each pair of coupled upper and lower solutions $\widetilde{U}_{s}, \widehat{U}_{s}$ of problems (1)-(3) is ones of (4). In what follows, we shall search a pair of constant solutions of (4), which is called upper and lower solutions of (4). 
Lemma 1. For constant $l_{1}>0$ and $L_{1}>0$ and any sufficiently large constant $L_{j}>0$ and small constant $l_{j}>0(j=2,3)$, the coupled upper and lower solutions to (4) is a constant pair $\mathbf{L}=\left(L_{1}, L_{2}, L_{3}\right)$ and $\mathbf{l}=\left(l_{1}, l_{2}, l_{3}\right)$.

Proof. Obviously, the constant pair $\mathbf{L}=\left(L_{1}, L_{2}, L_{3}\right)$ and $\mathbf{l}=$ $\left(l_{1}, l_{2}, l_{3}\right)$ satisfy the homogeneous Neumann boundary conditions (2). Further, by means of condition $\left(H_{1}\right)$, we can confirm that the differential inequalities in (4) can also be satisfied by the constant pair $\mathbf{L}$ and $\mathbf{l}$ if

$$
\begin{aligned}
\lambda-d L_{1}-g\left(L_{1}, l_{3}\right) & \leq \lambda-d L_{1} \leq 0, \\
g\left(L_{1}, L_{3}\right)-\delta L_{2} & \leq \eta L_{1} L_{3}-\delta L_{2} \leq 0, \\
p L_{2}-c L_{3} & \leq 0 \\
\lambda-d l_{1}-g\left(l_{1}, L_{3}\right) & \geq \lambda-d l_{1}-\eta l_{1} L_{3}=\lambda-\left(d+\eta L_{3}\right) l_{1} \geq 0, \\
g\left(l_{1}, l_{3}\right)-\delta l_{2} & \geq g_{v}\left(l_{1}, l_{3}\right) l_{3}-\delta l_{2} \geq 0, \\
p l_{2}-c l_{3} & \geq 0,
\end{aligned}
$$

and then, the above inequalities can be fulfilled for constants $l_{1}$ and $L_{1}$, sufficiently large $L_{j}$, and small $l_{j}(j=2,3)$ if

$$
\begin{gathered}
\frac{\lambda}{d} \leq L_{1}, \\
\frac{\lambda \eta L_{3}}{d \delta} \leq L_{2} \leq \frac{c L_{3}}{p}, \\
l_{1} \leq \frac{\lambda}{d+\eta L_{3}}, \\
l_{2} \leq \frac{g_{v}\left(l_{1}, l_{3}\right) l_{3}}{\delta}, \\
l_{3} \leq \frac{p l_{2}}{c} .
\end{gathered}
$$

To obtain the upper solution of (4), we only need to take $L_{1}=(\lambda / d)$ and sufficiently large $L_{2}$ and $L_{3}$ satisfying $\left(\lambda \eta L_{3} / d \delta\right) \leq L_{2} \leq\left(c L_{3} / p\right)$. Meanwhile, choosing sufficiently small $l_{3}, l_{2}=\left(c l_{3} / p\right)$, and $l_{1}$ satisfies $(c \delta / p) \leq g_{v}\left(l_{1}, l_{3}\right)$ and $l_{1} \leq\left(\lambda /\left(d+\eta L_{3}\right)\right)$, and we can also obtain the lower solution of (4). This means that the coupled upper and lower solutions of (4) are a constant pair $\mathbf{L}$ and $\mathbf{l}$. This completes the proof of Lemma 1 .
According to the results of Lemma 1 and method in $[6,7]$, we can conclude that problems (1)-(4) is well-posed similarly. That is, the solutions to problems (1)-(4) exist and are unique and bounded.

2.2. Equilibria. To describe that a single infected cell generates the average number of newly infected cells when the disease has just entered the body, we define the following basic reproduction number:

$$
\mathscr{R}_{0}=\frac{p}{c \delta} \frac{\partial g((\lambda / d), 0)}{\partial v},
$$

which is independent of spatial variety. Obviously, $E_{0}=((\lambda / d), 0,0)$ is always an infection-free equilibrium of model (1). Similar to the analysis and discussion in [27], together with the basic reproduction number $\mathscr{R}_{0}$, it is not hard to see that model (1) has only a positive equilibrium $E^{*}=\left(u^{*}, w^{*}, v^{*}\right)$ if $\mathscr{R}_{0}>1$. So, we have the results on the equilibria of model (1) as follows.

Theorem 1. The infection-free equilibrium $E_{0}=((\lambda / d), 0,0)$ of model (1) always exists. If $\mathscr{R}_{0}>1$, model (1) has only an infection equilibrium $E^{*}=\left(u^{*}, w^{*}, v^{*}\right)$.

\subsection{Stability Properties of Model (1)}

2.3.1. The Local Stability of Model (1). In this section, we shall investigate the local stability of model (1). Under the homogeneous Neumann boundary conditions, assume that $0=v_{0}<v_{1}<\cdots<v_{q}<\cdots$ be the eigenvalues of $-\Delta$ on $\Omega$ and for $q=0,1,2, \ldots$, and $E\left(\nu_{q}\right)$ be the eigenfunctions space corresponding to $\nu_{q}$ in $C^{1}(\Omega)$. Suppose that $E\left(\nu_{q}\right)$ has an orthonormal basis $\left\{\varphi_{q m}: m=1,2, \ldots, \operatorname{dim} E\left(\nu_{q}\right)\right\}$, $\mathbf{X}=\left[C^{1}(\Omega)\right]^{3}$, and $\mathbf{X}_{q m}=\left\{\mathbf{c} \varphi_{q m}: \mathbf{c} \in \mathbb{R}^{3}\right\}$. Thus, we obtain

$$
\begin{aligned}
\mathbf{X} & =\underset{q=1}{\oplus} \mathbf{X}_{q}, \\
\mathbf{X}_{q} & =\underset{m=1}{\operatorname{dim} E\left(v_{q}\right)} \mathbf{X}_{q m} .
\end{aligned}
$$

Linearizing system $(1)$ at the equilibrium $(\widetilde{u}, \widetilde{w}, \widetilde{v})$ is as follows:

$$
\left\{\begin{array}{l}
\frac{\partial u(x, t)}{\partial t}=D_{u}(\widetilde{u}) \Delta u-d u(x, t)-\frac{\partial g(\widetilde{u}, \widetilde{v})}{\partial u} u(x, t)-\frac{\partial g(\widetilde{u}, \widetilde{v})}{\partial v} v(x, t) \\
\frac{\partial w(x, t)}{\partial t}=D_{w}(\widetilde{w}) \Delta w+\frac{\partial g(\widetilde{u}, \widetilde{v})}{\partial u} u(x, t)+\frac{\partial g(\widetilde{u}, \widetilde{v})}{\partial v} v(x, t)-\delta w(x, t) \\
\frac{\partial v(x, t)}{\partial t}=D_{v}(\widetilde{v}) \Delta v+p w(x, t)-c v(x, t) .
\end{array}\right.
$$


Note that, under the linearization, $\mathbf{X}_{q}$ is an invariant for each $q=0,1,2, \ldots$. We let the expression of solution be $Q(x, t)=e^{\mu t} \psi(x)$, where $\psi(x) \in \mathbf{X}_{q}$ satisfies $-\Delta \psi=\nu_{q} \psi$. Thus, the characteristic equation of (12) is

$$
\begin{aligned}
& \left(\mu+D_{u}(\widetilde{u}) v_{q}+d+\frac{\partial g(\widetilde{u}, \widetilde{v})}{\partial u}\right) \\
& \cdot\left[\left(\mu+D_{w}(\widetilde{w}) v_{q}+\delta\right)\left(\mu+D_{v}(\widetilde{v}) v_{q}+c\right)-p \frac{\partial g(\widetilde{u}, \widetilde{v})}{\partial v}\right] \\
& +p \frac{\partial g(\widetilde{u}, \widetilde{v})}{\partial u} \frac{\partial g(\widetilde{u}, \widetilde{v})}{\partial v}=0 .
\end{aligned}
$$

When the equilibrium of model (1) is $E_{0}=((\lambda / d), 0,0)$, the characteristic equation (13) is changed into

$$
\left(\mu+D_{u}\left(\frac{\lambda}{d}\right) v_{q}+d\right)\left[(\mu+\delta)(\mu+c)-p \frac{\partial g((\lambda / d), 0)}{\partial v}\right]=0 .
$$

Obviously, equation (14) has a negative real root $\sigma=-D_{u}(\lambda / d) v_{q}-d$. We now discuss the transcendental equation as follows:

$$
(\mu+\delta)(\mu+c)-p \frac{\partial g((\lambda / d), 0)}{\partial v}=0
$$

Set

$$
H(\mu, q)=(\mu+\delta)(\mu+c)-p \frac{\partial g((\lambda / d), 0)}{\partial v} .
$$

Case 1. $\mathscr{R}_{0}<1$. By direct computation, we have

$$
H(0, q)=c \delta-p \frac{\partial g((\lambda / d), 0)}{\partial v}=c \delta\left(1-\mathscr{R}_{0}\right)>0,
$$

and for $\mu \geq 0$,

$$
\frac{\partial H(\mu, q)}{\partial \mu}=2 \mu+\delta+c>0
$$

So, there is only a negative real root for equation (14).
Assume that there be a complex root $\rho+i @$ with $\rho \geq 0$, then we can conclude that

$$
\begin{gathered}
\left|\frac{\mu}{\delta}+1\right|>1, \\
\left|\frac{\mu}{c}+1\right|>1, \\
\frac{p}{c \delta} \frac{\partial g((\lambda / d), 0)}{\partial v}=\mathscr{R}_{0}<1,
\end{gathered}
$$

which implies

$$
\left|\frac{\mu}{\delta}+1\right|\left|\frac{\mu}{c}+1\right|>\mathscr{R}_{0}=\frac{p}{c \delta} \frac{\partial g((\lambda / d), 0)}{\partial v} .
$$

This contradicts to equation (15). Then, all roots of equation (14) have no positive real parts yet. Thus, the infection-free equilibrium $E_{0}$ is locally asymptotically stable if $\mathscr{R}_{0}<1$.

Case 2. $\mathscr{R}_{0}>1$. Obviously, we need to only consider the space $\mathbf{X}_{0}$ corresponding to $v_{0}=0$ when $q=0$. By direct computation, we get

$$
\begin{aligned}
H(0,0) & =c \delta\left(1-\mathscr{R}_{0}\right)<0, \\
\lim _{\mu \longrightarrow+\infty} H(\mu, 0) & =+\infty .
\end{aligned}
$$

This implies that the equation $H(\mu, 0)=0$ must hold for some $\mu_{0}>0$. Thus, there exists at least one positive root of equation (14). So, the infection-free equilibrium $E_{0}$ is not stable for $\mathscr{R}_{0}>1$. Then, we have the following results.

Theorem 2. The infection-free equilibrium $E_{0}$ of model (1) is locally asymptotically stable if $\mathscr{R}_{0}<1$. However, $E_{0}$ is unstable if $\mathscr{R}_{0}>1$.

When the equilibrium of model (1) is $E^{*}=\left(u^{*}, w^{*}, v^{*}\right)$, the characteristic equation (13) is changed into

$$
\mu^{3}+A \mu^{2}+B \mu+C=0,
$$

where

$$
\begin{aligned}
& A=D_{u}\left(u^{*}\right) v_{q}+D_{w}\left(w^{*}\right) v_{q}+D_{v}\left(v^{*}\right) v_{q}+d+\delta+c+\frac{\partial g\left(u^{*}, v^{*}\right)}{\partial u} \\
& B=\left(D_{u}\left(u^{*}\right) v_{q}+d+\frac{\partial g\left(u^{*}, v^{*}\right)}{\partial u}\right)\left(D_{w}\left(w^{*}\right) v_{q}+D_{v}\left(v^{*}\right) v_{q}+\delta+c\right)+\left(D_{w}\left(w^{*}\right) v_{q}+\delta\right)\left(D_{v}\left(v^{*}\right) v_{q}+c\right)-p \frac{\partial g\left(u^{*}, v^{*}\right)}{\partial v} \\
& C=\left(D_{u}\left(u^{*}\right) v_{q}+d+\frac{\partial g\left(u^{*}, v^{*}\right)}{\partial u}\right)\left(\left(D_{w}\left(w^{*}\right) v_{q}+\delta\right)\left(D_{v}\left(v^{*}\right) v_{q}+c\right)-p \frac{\partial g\left(u^{*}, v^{*}\right)}{\partial v}\right)+p \frac{\partial g\left(u^{*}, v^{*}\right)}{\partial u} \frac{\partial g\left(u^{*}, v^{*}\right)}{\partial v}
\end{aligned}
$$

It is easy to see that $A>0$. From the condition $\left(H_{1}\right)$, together with $g\left(u^{*}, v^{*}\right)=\delta w^{*}$ and $p w^{*}=c v^{*}$, we get

$$
p \frac{\partial g\left(u^{*}, v^{*}\right)}{\partial v} \leq \frac{p g\left(u^{*}, v^{*}\right)}{v^{*}}=\frac{p \delta w^{*}}{\left(p w^{*} / c\right)}=c \delta,
$$


which yields $B>0$ and $C>0$. By direct computation, we have that

$$
\begin{aligned}
A B-C= & {\left[D_{u}\left(u^{*}\right) v_{q}+D_{w}\left(w^{*}\right) v_{q}+D_{v}\left(v^{*}\right) v_{q}+d+\delta+\frac{\partial g\left(u^{*}, v^{*}\right)}{\partial u}\right]\left(D_{u}\left(u^{*}\right) v_{q}+d+\frac{\partial g\left(u^{*}, v^{*}\right)}{\partial u}\right) } \\
& \cdot\left(D_{w}\left(w^{*}\right) v_{q}+D_{v}\left(v^{*}\right) v_{q}+\delta+c\right)+c\left(D_{u}\left(u^{*}\right) v_{q}+d\right)\left(c+D_{w}\left(w^{*}\right) v_{q}+D_{v}\left(v^{*}\right) v_{q}+\delta\right) \\
& +c \frac{\partial g\left(u^{*}, v^{*}\right)}{\partial u}\left(D_{w}\left(w^{*}\right) v_{q}+D_{v}\left(v^{*}\right) v_{q}+c\right)+\frac{\partial g\left(u^{*}, v^{*}\right)}{\partial u}\left(c \delta-p \frac{\partial g\left(u^{*}, v^{*}\right)}{\partial v}\right)>0 .
\end{aligned}
$$

So, we can see that all eigenvalues of (22) have no positive real parts by the Routh-Hurwitz criterion. Therefore, $E^{*}$ is locally asymptotically stable for $\mathscr{R}_{0}>1$. Thus, we obtain the following results.

Theorem 3. The infection equilibrium $E^{*}$ of model (1) is locally asymptotically stable if $\mathscr{R}_{0}>1$.

2.3.2. The Global Stability of Model (1). In this section, by constructing appropriate Lyapunov functionals, we continue to investigate the global stability of model (1). To this end, we need the following lemma.
Lemma 2. Under homogeneous Neumann boundary condition, for a density-dependent diffusion problem, we can obtain the following result:

$$
\int_{\Omega} \frac{1}{g(u)} \nabla \cdot(D(u) \nabla u) \mathrm{d} x=\int_{\Omega} \frac{D(u) g^{\prime}(u)\|\nabla u\|^{2}}{g^{2}(u)} \mathrm{d} x,
$$

where $\Omega$ is a bounded domain in $\mathbf{R}^{n}$ and its boundary $\partial \Omega$ is smooth.

Proof. According to the homogeneous Neumann boundary condition, we get

$$
\begin{aligned}
\int_{\Omega} \frac{1}{g(u)-} \nabla \cdot(D(u) \nabla u) \mathrm{d} x= & \int_{\Omega} \frac{1}{g(u)} D^{\prime}(u)\|\nabla u\|^{2} \mathrm{~d} x+\int_{\Omega} \frac{1}{g(u)} D(u) \Delta u \mathrm{~d} x \\
= & \int_{\Omega} \frac{1}{g(u)} D^{\prime}(u)\|\nabla u\|^{2} \mathrm{~d} x+\int_{\partial \Omega} \frac{1}{g(u)} D(u) \frac{\partial u}{\partial \nu} \mathrm{d} s-\int_{\Omega} D(u)\left(\nabla\left(\frac{1}{g(u)}\right) \cdot \nabla u\right) \mathrm{d} x \\
& -\int_{\Omega} \frac{1}{g(u)} D^{\prime}(u)\|\nabla u\|^{2} \mathrm{~d} x=\int_{\Omega} \frac{D(u) g^{\prime}(u)\|\nabla u\|^{2}}{g^{2}(u)} \mathrm{d} x .
\end{aligned}
$$

This completes the proof of Lemma 2.

Remark 1. Choosing $g(u)=1, u$, and $(1 / u)$ in Lemma 2, respectively, we can obtain that

(i) $\int_{\Omega} \nabla \cdot(D(u) \nabla u) \mathrm{d} x=0$;

(ii) $\int_{\Omega}(1 / u) \nabla \cdot(D(u) \nabla u) \mathrm{d} x=\int_{\Omega}\left(D(u)\|\nabla u\|^{2} / u^{2}\right) \mathrm{d} x$;

(iii) $\int_{\Omega} u \nabla \cdot(D(u) \nabla u) \mathrm{d} x=-\int_{\Omega} D(u)\|\nabla u\|^{2} \mathrm{~d} x$;

which implies that Lemma 2 generalized the results in $[6,7]$.
Firstly, we discuss the global asymptotic stability of the infection-free equilibrium $E_{0}$ of model (3).

Theorem 4. If $\mathscr{R}_{0} \leq 1$, then the infection-free equilibrium $E_{0}$ of model (3) is globally asymptotically stable.

Proof. Define the following Lyapunov functional:

$$
V(t)=\int_{\Omega}\left[w(x, t)+\frac{\delta}{p} v(x, t)\right] \mathrm{d} x .
$$


Through calculating the derivative of $V(t)$ about time, we can get

$$
\begin{aligned}
\frac{\mathrm{d} V(t)}{\mathrm{d} t} & =\int_{\Omega}\left[\frac{\partial w}{\partial t}+\frac{\delta}{p} \frac{\partial v}{\partial t}\right] \mathrm{d} x \\
& =\int_{\Omega}\left[\nabla \cdot(D(w) \nabla w)+g(u(x, t), v(x, t))-\delta w(x, t)+\frac{\delta}{p} \nabla \cdot(D(v) \nabla v)+\delta w(x, t)-\frac{c \delta}{p} v(x, t)\right] \mathrm{d} x \\
& =\int_{\Omega}\left[g(u(x, t), v(x, t))-\frac{c \delta}{p} v(x, t)\right] \mathrm{d} x+\int_{\Omega} \nabla \cdot(D(w) \nabla w) \mathrm{d} x+\frac{\delta}{p} \int_{\Omega} \nabla \cdot(D(v) \nabla v) \mathrm{d} x .
\end{aligned}
$$

Note that $(\partial u / \partial t) \leq \lambda-d u$, then we have $\lim _{t \rightarrow \infty} u$ $(x, t) \leq(\lambda / d)$, which means that all omega limit points satisfy $u \leq(\lambda / d)$. So, we need to only consider solutions for which $u \leq(\lambda / d)$. Since $(\partial g / \partial u) \geq 0$, we can conclude the inequality $g(u, v) \leq g((\lambda / d), v)$. Under the condition $\left(H_{1}\right)$, together with the fact that $(g((\lambda / d), v) / v)$ is decreasing in $v$, we can obtain

$$
\begin{aligned}
g(u(x, t), v(x, t))-\frac{c \delta}{p} v(x, t) & =\frac{c \delta}{p} v\left(\frac{p}{c \delta} \frac{g(u, v)}{v}-1\right) \\
& \leq \frac{c \delta}{p} v\left(\frac{p}{c \delta} \frac{g((\lambda / d), v)}{v}-1\right) \leq \frac{c \delta}{p} v\left(\lim _{v \longrightarrow 0^{+}} \frac{p}{c \delta} \frac{g((\lambda / d), v)}{v}-1\right) \\
& =\frac{c \delta}{p} v\left(\frac{p}{c \delta} \frac{\partial g((\lambda / d), 0)}{\partial v}-1\right)=\frac{c \delta}{p} v\left(\mathscr{R}_{0}-1\right) .
\end{aligned}
$$

Meanwhile, from Remark 1, we get

$\int_{\Omega} \nabla \cdot(D(w) \nabla w) \mathrm{d} x=0=\frac{\delta}{p} \int_{\Omega} \nabla \cdot(D(v) \nabla v) \mathrm{d} x$.

Thus, we obtain

$$
\frac{\mathrm{d} V(t)}{\mathrm{d} t} \leq \frac{c \delta}{p}\left(\mathscr{R}_{0}-1\right) \int_{\Omega} v \mathrm{~d} x .
$$

So, we have that $(\mathrm{d} V(t) / \mathrm{d} t) \leq 0$ when $\mathscr{R}_{0} \leq 1$. Further, it is not hard to believe that $(\mathrm{d} V(t) / \mathrm{d} t) \leq 0$ leads to either $v=0$ or $\mathscr{R}_{0}=1$ and $u=(\lambda / d)$. It is easy to confirm that the singleton $\left\{E_{0}\right\}$ is just the largest compact invariant set in $\{(u, w, v) \mid(\mathrm{d} V(t) / \mathrm{d} t) \leq 0\}$. When $\mathscr{R}_{0} \leq 1$, we can know that the infection-free equilibrium $E_{0}$ of model (3) is globally asymptotically stable by the LaSalle's invariance principle. The proof of Theorem 4 is complete.

Next, we investigate the global asymptotic stability problem of the infection equilibrium $E^{*}$ of model (3). To prove the following theorem, we need the following hypothesis:

$$
\left(H_{3}\right)\left(\frac{v}{v^{*}}-\frac{g(u, v)}{g\left(u, v^{*}\right)}\right)\left(\frac{g(u, v)}{g\left(u, v^{*}\right)}-1\right) \geq 0, \quad \text { for all } u, v>0 \text {. }
$$

Theorem 5. Suppose $\left(H_{3}\right)$ is satisfied. If $\mathscr{R}_{0}>1$, then the infection equilibrium $E^{*}$ of model (3) is globally asymptotically stable.

Proof. Let $H(z)=z-1-\ln z, z>0$, and define

$$
\begin{aligned}
V(t)= & \int_{\Omega}\left\{u-u^{*}-\int_{u^{*}}^{u(x, t)} \frac{g\left(u^{*}, v^{*}\right)}{g\left(s, v^{*}\right)} \mathrm{d} s+w^{*} H\left(\frac{w}{w^{*}}\right)\right. \\
& \left.+\frac{\delta}{p} v^{*} H\left(\frac{v}{v^{*}}\right)\right\} \mathrm{d} x,
\end{aligned}
$$

which is called a Lyapunov functional. Obviously, 0 is the minimum value of function $H(z)$ at $z=1$. This leads to $H(z) \geq 0$. Meanwhile, 0 is also the global minimum of function $G: u \longrightarrow u-u^{*}-\int_{u^{*}}^{u}\left(g\left(u^{*}, v^{*}\right) / g\left(s, v^{*}\right)\right) \mathrm{d} s$ at $u=u^{*}$, which implies $G(u) \geq 0$ for any $x>0$. Therefore, for all $x \in \Omega$ and $t \geq 0, V(t) \geq 0$ with equality holding if and only if $\left(u(x, t) / u^{*}\right)=\left(w / w^{*}\right)=\left(v(x, t) / v^{*}\right)=1$.

Through calculating the derivative of $V(t)$ about time, we can get 


$$
\begin{aligned}
\frac{\mathrm{d} V(t)}{\mathrm{d} t}= & \int_{\Omega}\left\{\left(1-\frac{g\left(u^{*}, v^{*}\right)}{g\left(u, v^{*}\right)}\right) \frac{\partial u}{\partial t}+\left(1-\frac{w^{*}}{w}\right) \frac{\partial w}{\partial t}+\frac{\delta}{p}\left(1-\frac{v^{*}}{v}\right) \frac{\partial v}{\partial t}\right\} \mathrm{d} x \\
= & \int_{\Omega}\left\{\left(1-\frac{g\left(u^{*}, v^{*}\right)}{g\left(u, v^{*}\right)}\right)[\nabla \cdot(D(u) \nabla u)+\lambda-d u(x, t)-g(u(x, t), v(x, t))]\right. \\
& \left.+\left(1-\frac{w^{*}}{w}\right)[\nabla \cdot(D(w) \nabla w)+g(u(x, t), v(x, t))-\delta w(x, t)]+\frac{\delta}{p}\left(1-\frac{v^{*}}{v}\right)[\nabla \cdot(D(v) \nabla v)+p w(x, t)-c v(x, t)]\right\} \mathrm{d} x
\end{aligned}
$$

Note that $\lambda=d u^{*}+g\left(u^{*}, v^{*}\right), \delta w^{*}=g\left(u^{*}, v^{*}\right)$, and $c v^{*}=p w^{*}$. So, we have

$$
\begin{aligned}
& \frac{\mathrm{d} V(t)}{\mathrm{d} t}=\int_{\Omega}\left\{\left(1-\frac{g\left(u^{*}, v^{*}\right)}{g\left(u, v^{*}\right)}\right)\left(\nabla \cdot(D(u) \nabla u)+d u^{*}-d u(x, t)+g\left(u^{*}, v^{*}\right)-g(u(x, t), v(x, t))\right)\right. \\
& +\left(1-\frac{w^{*}}{w}\right)\left(\nabla \cdot(D(w) \nabla w)+g(u(x, t), v(x, t))-\frac{w(x, t)}{w^{*}} g\left(u^{*}, v^{*}\right)\right)+\frac{\delta}{p}\left(1-\frac{v^{*}}{v}\right)(\nabla \cdot(D(v) \nabla v)) \\
& \left.\left.+p\left(w(x, t)-\frac{v(x, t)}{v^{*}} w^{*}\right)\right)\right\} \mathrm{d} x \\
& =\int_{\Omega}\left\{d u^{*}\left(1-\frac{u}{u^{*}}\right)\left(1-\frac{g\left(u^{*}, v^{*}\right)}{g\left(u, v^{*}\right)}\right)+g\left(u^{*}, v^{*}\right)\left[\left(1-\frac{g\left(u^{*}, v^{*}\right)}{g\left(u, v^{*}\right)}\right)\left(1-\frac{g(u(x, t), v(x, t)}{g\left(u^{*}, v^{*}\right)}\right)\right.\right. \\
& \left.\left.+\left(1-\frac{w^{*}}{w}\right)\left(\frac{g(u(x, t), v(x, t))}{g\left(u^{*}, v^{*}\right)}-\frac{w(x, t)}{w^{*}}\right)+\left(1-\frac{v^{*}}{v}\right)\left(\frac{w(x, t)}{w^{*}}-\frac{v(x, t)}{v^{*}}\right)\right]\right\} \mathrm{d} x \\
& +\int_{\Omega}\left(1-\frac{g\left(u^{*}, v^{*}\right)}{g\left(u, v^{*}\right)}\right) \nabla \cdot(D(u) \nabla u) \mathrm{d} x+\int_{\Omega}\left(1-\frac{w^{*}}{w}\right) \nabla \cdot(D(w) \nabla w) \mathrm{d} x+\frac{\delta}{p} \int_{\Omega}\left(1-\frac{v^{*}}{v}\right) \nabla \cdot(D(v) \nabla v) \mathrm{d} x \\
& =\int_{\Omega}\left\{d u^{*}\left(1-\frac{u}{u^{*}}\right)\left(1-\frac{g\left(u^{*}, v^{*}\right)}{g\left(u, v^{*}\right)}\right)+g\left(u^{*}, v^{*}\right)\left[H\left(\frac{g(u(x, t), v(x, t))}{g\left(u(x, t), v^{*}\right)}\right)-H\left(\frac{g\left(u^{*}, v^{*}\right)}{g\left(u(x, t), v^{*}\right)}\right)\right.\right. \\
& -H\left(\frac{g(u(x, t), v(x, t))}{g\left(u^{*}, v^{*}\right)}\right)+H\left(\frac{g(u(x, t), v(x, t))}{g\left(u^{*}, v^{*}\right)}\right)-H\left(\frac{g(u(x, t), v(x, t)) w^{*}}{g\left(u^{*}, v^{*}\right) w(x, t)(x, t)}\right)-H\left(\frac{w(x, t)}{w^{*}}\right) \\
& \left.\left.+H\left(\frac{w(x, t)}{w^{*}}\right)-H\left(\frac{w(x, t) v^{*}}{v(x, t) w^{*}}\right)-H\left(\frac{v(x, t)}{v^{*}}\right)\right]\right\} \mathrm{d} x \\
& +\int_{\Omega}\left(1-\frac{g\left(u^{*}, v^{*}\right)}{g\left(u, v^{*}\right)}\right) \nabla \cdot(D(u) \nabla u) \mathrm{d} x+\int_{\Omega}\left(1-\frac{w^{*}}{w}\right) \nabla \cdot(D(w) \nabla w) \mathrm{d} x+\frac{\delta}{p} \int_{\Omega}\left(1-\frac{v^{*}}{v}\right) \nabla \cdot(D(v) \nabla v) \mathrm{d} x \\
& =\int_{\Omega}\left\{d u^{*}\left(1-\frac{u}{u^{*}}\right)\left(1-\frac{g\left(u^{*}, v^{*}\right)}{g\left(u, v^{*}\right)}\right)+g\left(u^{*}, v^{*}\right)\left[H\left(\frac{g(u(x, t), v(x, t))}{g\left(u(x, t), v^{*}\right)}\right)-H\left(\frac{g\left(u^{*}, v^{*}\right)}{g\left(u(x, t), v^{*}\right)}\right)\right.\right. \\
& \left.\left.-H\left(\frac{g(u(x, t), v(x, t)) w^{*}}{g\left(u^{*}, v^{*}\right) w(x, t)}\right)-H\left(\frac{w(x, t) v^{*}}{v(x, t) w^{*}}\right)-H\left(\frac{v(x, t)}{v^{*}}\right)\right]\right\} \mathrm{d} x \\
& +\int_{\Omega}\left(1-\frac{g\left(u^{*}, v^{*}\right)}{g\left(u, v^{*}\right)}\right) \nabla \cdot(D(u) \nabla u) \mathrm{d} x+\int_{\Omega}\left(1-\frac{w^{*}}{w}\right) \nabla \cdot(D(w) \nabla w) \mathrm{d} x+\frac{\delta}{p} \int_{\Omega}\left(1-\frac{v^{*}}{v}\right) \nabla \cdot(D(v) \nabla v) \mathrm{d} x .
\end{aligned}
$$


From the hypothesis $\left(H_{1}\right)$, we can know that $g\left(u, v^{*}\right)$ is increasing in $u$ strictly. Hence, we have

$$
\left(1-\frac{u}{u^{*}}\right)\left(1-\frac{g\left(u^{*}, v^{*}\right)}{g\left(u, v^{*}\right)}\right) \leq 0,
$$

and $\left(1-\left(u / u^{*}\right)\right)\left(1-\left(g\left(u^{*}, v^{*}\right) / g\left(u, v^{*}\right)\right)\right)$ equals 0 only if $u=u^{*}$. Based on the assumption $\left(H_{3}\right)$, we can conclude that $\left(g(u(x, t), v(x, t)) / g\left(u(x, t), v^{*}\right)\right) \quad$ lies between 1 and $\left(v(x, t) / v^{*}\right)$. Thus, by the fact that $H(z)$ is a monotone function on each side of 1 , we can obtain that $H\left(g(u(x, t), v(x, t)) / g\left(u(x, t), v^{*}\right)\right) \leq H\left(v(x, t) / v^{*}\right)$.

Therefore,

$$
\begin{aligned}
\frac{\mathrm{d} V(t)}{\mathrm{d} t} \leq & -g\left(u^{*}, v^{*}\right) \int_{\Omega}\left[H\left(\frac{g\left(u^{*}, v^{*}\right)}{g\left(u, v^{*}\right)}\right)+H\left(\frac{w(x, t) v^{*}}{v(x, t) w^{*}}\right)+H\left(\frac{g(u(x, t), v(x, t)) w^{*}}{g\left(u^{*}, v^{*}\right) w(x, t)}\right) \mathrm{d} x\right] \\
& +\int_{\Omega}\left(1-\frac{g\left(u^{*}, v^{*}\right)}{g\left(u, v^{*}\right)}\right) \nabla \cdot(D(u) \nabla u) \mathrm{d} x+\int_{\Omega}\left(1-\frac{w^{*}}{w}\right) \nabla \cdot(D(w) \nabla w) \mathrm{d} x+\frac{\delta}{p} \int_{\Omega}\left(1-\frac{v^{*}}{v}\right) \nabla \cdot(D(v) \nabla v) \mathrm{d} x .
\end{aligned}
$$

From Lemma 2 and Remark 1, it is easy to conclude that

$$
\frac{\mathrm{d} V(t)}{\mathrm{d} t} \leq 0 \text {. }
$$

Hence, $E^{*}$ is stable and $(\mathrm{d} V(t) / \mathrm{d} t)=0$ iff $u=u^{*}$, $w=w^{*}$, and $v=v^{*}$. It is easy to confirm that the singleton $\left\{E^{*}\right\}$ is just the largest compact invariant set in $\{(u, w, v) \mid(\mathrm{d} V(t) / \mathrm{d} t)=0\}$. According to the LaSalle's invariance principle, we can know that the infection equilibrium $E^{*}$ of model (3) is globally asymptotically stable when $\mathscr{R}_{0}>1$. This completes the proof of Theorem 5 .

\section{Multistrain Viral Infection Model}

In this section, we shall begin to study the multi-strain viral infection model (7) with Neumann boundary conditions:

$$
\begin{aligned}
& D_{u}(u) \frac{\partial u}{\partial \nu}=0, \\
& D_{w_{i}}\left(w_{i}\right) \frac{\partial w_{i}}{\partial \nu}=0, \\
& D_{v_{i}}\left(v_{i}\right) \frac{\partial v_{i}}{\partial \nu}=0, \\
& \text { on } \partial \Omega \times(0,+\infty),
\end{aligned}
$$

and initial conditions

$$
\begin{aligned}
u(x, 0) & =\phi_{1}(x) \geq 0, \\
w_{i}(x, 0) & =\phi_{2, i}(x) \geq 0, \\
v_{i}(x, 0) & =\phi_{3, i}(x) \geq 0, \\
x & \in \bar{\Omega} .
\end{aligned}
$$

Suppose $\Omega$ is a bounded domain in $\mathbb{R}^{n}$ and its boundary $\partial \Omega$ is smooth, then the outward normal derivative on $\partial \Omega$ is denoted by $\partial \nu$. The nonnegative functions $\phi_{1}(x, 0)$ and $\phi_{r, i}(x, 0) \quad(r=2,3, i=1,2, \ldots, n)$ are Hölder continuous and satisfy $\left(\partial \phi_{1} / \partial \nu\right)=0$ and $\left(\partial \phi_{r, i} / \partial \nu\right)=0$ on $\partial \Omega$, respectively. Moreover, we can get the steady-state system of model (7) as follows:

$$
\left\{\begin{array}{l}
-\nabla \cdot\left(D_{u}(u) \nabla u\right)=\lambda-d u(x, t)-\sum_{i=1}^{n} g_{i}\left(u(x, t), v_{i}(x, t)\right), \\
-\nabla \cdot\left(D_{w_{i}}\left(w_{i}\right) \nabla w_{i}\right)=g_{i}\left(u(x, t), v_{i}(x, t)\right)-\delta_{i} w_{i}(x, t), \\
-\nabla \cdot\left(D_{v_{i}}\left(v_{i}\right) \nabla v_{i}\right)=p_{i} w_{i}(x, t)-c_{i} v_{i}(x, t), \quad i=1,2, \ldots, n,
\end{array}\right.
$$

where $D_{u}(u)=u^{m_{1}}, D_{w_{i}}\left(w_{i}\right)=w_{i}^{m_{2, i}}$, and $D_{v_{i}}\left(v_{i}\right)=v_{i}^{m_{3, i}}$ are the density-dependent diffusion coefficients for $m_{1}>0$ and $m_{r, i}>0, r=2,3, i=1,2, \ldots, n$.

3.1. Well Posedness. In this section, we give out some definitions and results first, which can help us to prove that the solutions to problems (7) and (42) exist and are unique and bounded. Similar to the definition method in $[6,7]$, we can definite that two pairs of functions $\widetilde{U}=\left(\widetilde{u}, \widetilde{w}_{i}, \widetilde{v}_{i}\right), \widehat{U}=\left(\widehat{u}, \widehat{w}_{i}, \widehat{v}_{i}\right)$ in $C(\bar{\Omega} \times[0, T]) \cap C^{1,2}(\Omega \times$ $[0, T])$ and $\tilde{U}_{s}=\left(\widetilde{u}_{s}, \widetilde{w}_{i, s}, \widetilde{v}_{i, s}\right), \widehat{U}_{s}=\left(\widehat{u}_{s}, \widehat{w}_{i, s}, \widehat{v}_{i, s}\right)$ in $C(\bar{\Omega} \times$ $[0, T]) \cap C^{1,2}(\Omega \times[0, T])$ are called coupled upper and lower solutions to system (7) and to system (42), respectively. Then, we can easily conclude that each pair of coupled upper and lower solutions $\widetilde{U}_{s}$ and $\widehat{U}_{s}$ of problems (7) is also ones of (42). In what follows, we shall search a pair of constant solutions of (42), which is called upper and lower solutions of (42).

Lemma 3. For constant $l_{1}>0$ and $L_{1}>0$ and any sufficiently large constant $L_{2, i}>0$ and $L_{3, i}>0$ and small constant $l_{2, i}>0$ and $l_{3, i}>0, i=1,2, \ldots, n$, the coupled upper and lower solutions to (42) are a constant pair $\mathbf{L}=\left(L_{1}, L_{2, i}, L_{3, i}\right)$ and $\mathbf{l}=\left(l_{1}, l_{2, i}, l_{3, i}\right)$. 
Proof. Obviously, the constant pair $\mathbf{L}=\left(L_{1}, L_{2, i}, L_{3, i}\right)$ and $\mathbf{l}=\left(l_{1}, l_{2, i}, l_{3, i}\right)$ satisfy the homogeneous Neumann boundary conditions (40). Further, by means of the condition $\left(H_{1}\right)$, we can confirm that the differential inequalities in (42) can also satisfied by a constant pair $\mathbf{L}$ and 1 if

$$
\begin{gathered}
\lambda-d L_{1}-\sum_{i=1}^{n} g_{i}\left(L_{1}, l_{3, i}\right) \leq \lambda-d L_{1} \leq 0 \\
g_{i}\left(L_{1}, L_{3, i}\right)-\delta L_{2, i} \leq \eta_{i} L_{1} L_{3, i}-\delta L_{2, i} \leq 0 \\
p_{i} L_{2, i}-c_{i} L_{3, i} \leq 0 \\
\lambda-d l_{1}-\sum_{i=1}^{n} g_{i}\left(l_{1}, L_{3, i}\right) \geq \lambda-d l_{1} \\
-\sum_{i=1}^{n} \eta_{i} l_{1} L_{3, i}=\lambda-\left(d+\sum_{i=1}^{n} \eta_{i} L_{3, i}\right) l_{1} \geq 0 \\
g_{i}\left(l_{1}, l_{3, i}\right)-\delta l_{2, i} \geq g_{i v}\left(l_{1}, l_{3, i}\right) l_{3, i}-\delta l_{2, i} \geq 0 \\
p_{i} l_{2, i}-c_{i} l_{3, i} \geq 0 .
\end{gathered}
$$

Then, the above inequalities can be fulfilled for constants $l_{1}$ and $L_{1}$, sufficiently large $L_{2, i}$ and $L_{3, i}$, and small $l_{2, i}$ and $l_{3, i}(i=1,2, \ldots, n)$ if

$$
\begin{gathered}
\frac{\lambda}{d} \leq L_{1}, \\
\frac{\lambda \eta_{i} L_{3, i}}{d \delta_{i}} \leq L_{2, i} \leq \frac{c L_{3, i}}{p}, \\
l_{1} \leq \frac{\lambda}{d+\sum_{i=0}^{n} \eta_{i} L_{3, i}}, \\
l_{2, i} \leq \frac{g_{i v}\left(l_{1}, l_{3, i}\right) l_{3, i}}{\delta_{i}}, \\
l_{3, i} \leq \frac{p_{i} l_{2, i}}{c_{i}} .
\end{gathered}
$$

To obtain the upper solution of (42), we only need to take $L_{1}=(\lambda / d)$ and sufficiently large $L_{2}$ and $L_{3}$ satisfying $\left(\lambda \eta_{i} L_{3, i} / d \delta_{i}\right) \leq L_{2, i} \leq\left(c L_{3, i} / p\right)$. Meanwhile, choosing sufficiently small $l_{3, i}, \quad l_{2, i}=\left(c_{i} l_{3, i} / p_{i}\right)$, and $l_{1}$ satisfies $\left(c_{i} \delta_{i} / p_{i}\right) \leq g_{i v}\left(l_{1}, l_{3, i}\right)$ and $l_{1} \leq\left(\lambda /\left(d+\sum_{i=1}^{n} \eta_{i}\right)\right)$, and we can also obtain the lower solution of (42). This means that the coupled upper and lower solutions of (42) are a constant pair $\mathbf{L}$ and 1. This completes the proof of Lemma 3.

According to the results of Lemma 3 and method in $[6,7]$, we can conclude that problems (7) and (42) is wellposed similarly. That is, the solutions to problems (7) and (42) exist and are unique and bounded.
3.2. Competitive Exclusion. To describe a single-strain $i$-infected cell which generates the average number of newly infected cells when the disease has just entered the body, we define

$$
\mathscr{R}_{i}=\frac{p_{i}}{c_{i} \delta_{i}} \frac{\partial g_{i}((\lambda / d), 0)}{\partial v_{i}},
$$

which is called the basic reproduction number of strain $i$ in model (7). Obviously, $E_{0}^{n}=((\lambda / d), \mathbf{0 , 0})$ is always an infection-free steady state of system (7), where $\mathbf{0}$ is an $n$-dimensional vector of zeros. Similar to the analysis and discussion in [27], together with the basic reproduction number $\mathscr{R}_{i}$, it is not hard to see that for each strain $i$, model (7) has only a corresponding single-strain steady state as follows:

$$
E_{i}=\left(u^{*}, 0, \ldots, 0, w_{i}^{*}, 0, \ldots, 0, v_{i}^{*}, 0, \ldots, 0\right),
$$

iff $\mathscr{R}_{i}>1$ and it satisfies

$$
\left\{\begin{array}{l}
\lambda-d u(x, t)-g_{i}\left(u(x, t), v_{i}(x, t)\right)=0, \\
g_{i}\left(u(x, t), v_{i}(x, t)\right)-\delta_{i} w_{i}(x, t)=0, \\
p_{i} w_{i}(x, t)-c_{i} v_{i}(x, t)=0 .
\end{array}\right.
$$

Here, we have to point out that the nonzero components $w_{i}^{*}$ is in position $i+1$ and $v_{i}^{*}$ are in position $n+i+1$.

Now, we begin to investigate the competitive exclusion problem of model (7). From the results in [36], to discuss the competitive exclusion problem, we only need to discuss the globally asymptotical stability of steady state $E_{1}$ of strain one. Next, we shall verify that the globally asymptotical stability of the infected steady state $E_{1}$ of strain one by constructing an appropriate Lyapunov functional. Here, we also assume that for $i=1,2, \ldots, n, g_{i}\left(u, v_{i}\right)$ satisfies the condition $\left(H_{1}\right)$ and $\eta_{k}<\eta_{1}, k=2, \ldots, n$. Moreover, we need the following condition:

$$
\left(H_{4}\right) \eta_{k} \frac{p_{k}}{c_{k} \delta_{k}} \leq \frac{d}{\lambda} \frac{p_{1}}{c_{1} \delta_{1}} \min _{u \in(0,(\lambda / d))} \frac{\partial g_{1}\left(u, v_{1}^{*}\right)}{\partial v_{1}} .
$$

Theorem 6. Suppose $\left(H_{3}\right)$ and $\left(H_{4}\right)$ are satisfied. For $k=2, \ldots, n$, let $\mathscr{R}_{1}>1$ and $\mathscr{R}_{1}>\mathscr{R}_{k}$ hold. Then, the singlestrain steady state $E_{1}$ of model (7) is globally asymptotically stable.

Proof. Let $H(z)=z-1-\ln z, z>0$ and define

$$
\begin{aligned}
V(t)= & \int_{\Omega}\left\{u-u_{1}^{*}-\int_{u_{1}^{*}}^{u(x, t)} \frac{g_{1}\left(u_{1}^{*}, v_{1}^{*}\right)}{g_{1}\left(s, v_{1}^{*}\right)} \mathrm{d} s+w_{1}^{*} H\left(\frac{w_{1}}{w_{1}^{*}}\right)\right. \\
& \left.+\frac{\delta_{1}}{p_{1}} v_{1}^{*} H\left(\frac{v_{1}}{v_{1}^{*}}\right)+\sum_{k=2}^{n}\left(w_{k}+\frac{\delta_{k}}{p_{k}} v_{k}\right)\right\} \mathrm{d} x,
\end{aligned}
$$

which is call a Lyapunov functional. Obviously, 0 is the minimum value of function $H(z)$ at $z=1$. This leads to 

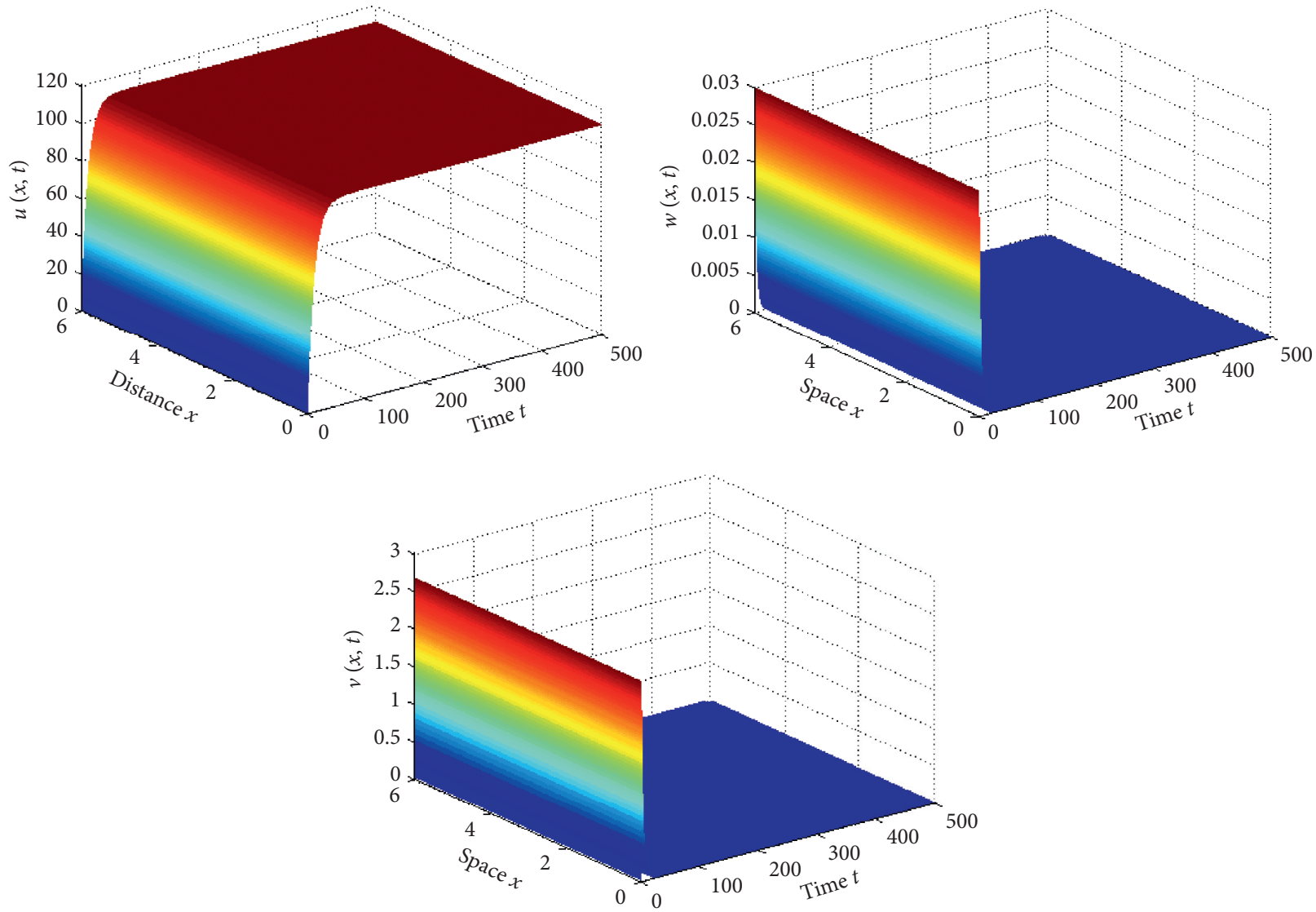

Figure 1: Choosing $m_{1}=m_{2}=m_{3}=2, \lambda=10, d=0.09, \beta=0.0005, \delta=0.4, c=2.4, p=300, a=0.5$, and $b=0.4$, we have $\mathscr{R}_{0}=0.3070<1$ and the infection-free equilibrium $E_{0}=(111.1111,0,0)$ of model (59) is globally asymptotically stable.

$H(z) \geq 0$. Meanwhile, the 0 is also the global minimum of function $G: u \longrightarrow u-u_{1}^{*}-\int_{u^{*}}^{u}\left(g_{1}\left(u_{1}^{*}, v_{1}^{*}\right) / g_{1}\left(s, v_{1}^{*}\right)\right) \mathrm{d} s$ at $u=u_{1}^{*}$, which implies $G(u) \geq 0$ for any $x>0$. Therefore, $V(t) \geq 0$ with equality holding if and only $\left(u(x, t) / u_{1}^{*}\right)=$ $\left(w_{1}(x, t) / w_{1}^{*}\right)=\left(v_{1}(x, t) / v_{1}^{*}\right)=1$ and $w_{k}=v_{k}=0(k=2$, $\ldots, n)$ for all $x \in \Omega$ and $t \geq 0$.

Through calculating the derivative of $V(t)$ about time, we get

$$
\begin{aligned}
\frac{\mathrm{d} V(t)}{\mathrm{d} t}= & \int_{\Omega}\left\{\left(1-\frac{g_{1}\left(u_{1}^{*}, v_{1}^{*}\right)}{g_{1}\left(u, v_{1}^{*}\right)}\right) \frac{\partial u}{\partial t}+\left(1-\frac{w_{1}^{*}}{w_{1}}\right) \frac{\partial w_{1}}{\partial t}+\frac{\delta_{1}}{p_{1}}\left(1-\frac{v_{1}^{*}}{v_{1}}\right) \frac{\partial v_{1}}{\partial t}+\sum_{k=2}^{n}\left(\frac{\partial w_{k}}{\partial t}+\frac{\delta_{k}}{p_{k}} \frac{\partial v_{k}}{\partial t}\right)\right\} \mathrm{d} x \\
= & \int_{\Omega}\left\{\left(1-\frac{g_{1}\left(u_{1}^{*}, v_{1}^{*}\right)}{g_{1}\left(u, v_{1}^{*}\right)}\right)\left[\nabla \cdot(D(u) \nabla u)+\lambda-d u(x, t)-g_{1}\left(u(x, t), v_{1}(x, t)\right)\right]\right. \\
& +\left(1-\frac{w_{1}^{*}}{w_{1}}\right)\left[\nabla \cdot\left(D\left(w_{1}\right) \nabla w_{1}\right)+g_{1}\left(u(x, t), v_{1}(x, t)\right)-\delta_{1} w_{1}(x, t)\right] \\
& +\frac{\delta_{1}}{p_{1}}\left(1-\frac{v_{1}^{*}}{v_{1}}\right)\left[\nabla \cdot\left(D\left(v_{1}\right) \nabla v_{1}\right)+p_{1} w_{1}(x, t)-c_{1} v_{1}(x, t)\right]-\left(1-\frac{g_{1}\left(u_{1}^{*}, v_{1}^{*}\right)}{g_{1}\left(u, v_{1}^{*}\right)}\right) \sum_{k=2}^{n} g_{k}\left(u(x, t), v_{k}(x, t)\right) \\
& \left.+\sum_{k=2}^{n}\left[\nabla \cdot\left(D\left(w_{k}\right) \nabla w_{k}\right)+g_{k}\left(u(x, t), v_{k}(x, t)\right)-\delta_{k} w_{k}(x, t)\right] \sum_{k=2}^{n} \frac{\delta_{k}}{p_{k}}\left[\nabla \cdot\left(D\left(v_{k}\right) \nabla v_{k}\right)+p_{k} w_{k}(x, t)-c_{k} v_{k}(x, t)\right]\right\} \mathrm{d} x .
\end{aligned}
$$


Note that $\lambda=d u_{1}^{*}+g_{1}\left(u_{1}^{*}, v_{1}^{*}\right), \delta_{1} w_{1}^{*}=g_{1}\left(u_{1}^{*}, v_{1}^{*}\right)$, and $c_{1} v_{1}^{*}=p_{1} w_{1}^{*}$. So, we have

$$
\begin{aligned}
& \frac{\mathrm{d} V(t)}{\mathrm{d} t}=\int_{\Omega}\left\{\left(1-\frac{g_{1}\left(u_{1}^{*}, v_{1}^{*}\right)}{g_{1}\left(u, v_{1}^{*}\right)}\right)\left(\nabla \cdot(D(u) \nabla u)+d u_{1}^{*}-d u(x, t)+g_{1}\left(u_{1}^{*}, v_{1}^{*}\right)-g_{1}\left(u(x, t), v_{1}(x, t)\right)\right)\right. \\
& +\left(1-\frac{w_{1}^{*}}{w_{1}}\right)\left(\nabla \cdot\left(D\left(w_{1}\right) \nabla w_{1}\right)+g_{1}\left(u(x, t), v_{1}(x, t)\right)-\frac{w_{1}(x, t)}{w_{1}^{*}} g_{1}\left(u_{1}^{*}, v_{1}^{*}\right)\right) \\
& +\frac{\delta_{1}}{p_{1}}\left(1-\frac{v_{1}^{*}}{v_{1}}\right)\left(\nabla \cdot\left(D\left(v_{1}\right) \nabla v_{1}\right)+p_{1}\left(w_{1}(x, t)-\frac{v_{1}(x, t)}{v_{1}^{*}} w_{1}^{*}\right)\right) \\
& +\sum_{k=2}^{n}\left[\nabla \cdot\left(D\left(w_{k}\right) \nabla w_{k}\right)+\frac{g_{1}\left(u_{1}^{*}, v_{1}^{*}\right)}{g_{1}\left(u, v_{1}^{*}\right)} g_{k}\left(u(x, t), v_{k}(x, t)\right)-\delta_{k} w_{k}(x, t)\right] \\
& \left.+\sum_{k=2}^{n} \frac{\delta_{k}}{p_{k}}\left[\nabla \cdot\left(D\left(v_{k}\right) \nabla v_{k}\right)+p_{k} w_{k}(x, t)-c_{k} v_{k}(x, t)\right]\right\} \mathrm{d} x \\
& =\int_{\Omega}\left\{d u_{1}^{*}\left(1-\frac{u}{u_{1}^{*}}\right)\left(1-\frac{g_{1}\left(u_{1}^{*}, v_{1}^{*}\right)}{g_{1}\left(u, v^{*}\right)}\right)+g_{1}\left(u_{1}^{*}, v_{1}^{*}\right)\left[\left(1-\frac{g_{1}\left(u_{1}^{*}, v_{1}^{*}\right)}{g_{1}\left(u, v_{1}^{*}\right)}\right)\left(1-\frac{g_{1}\left(u(x, t), v_{1}(x, t)\right)}{g_{1}\left(u_{1}^{*}, v_{1}^{*}\right)}\right)\right.\right. \\
& \left.\left.+\left(1-\frac{w_{1}^{*}}{w_{1}}\right)\left(\frac{g_{1}\left(u(x, t), v_{1}(x, t)\right)}{g\left(u_{1}^{*}, v_{1}^{*}\right)}-\frac{w_{1}(x, t)}{w_{1}^{*}}\right)+\left(1-\frac{v_{1}^{*}}{v_{1}}\right)\left(\frac{w_{1}(x, t)}{w_{1}^{*}}-\frac{v_{1}(x, t)}{v_{1}^{*}}\right)\right]\right\} \mathrm{d} x \\
& +\sum_{k=2}^{n} \int_{\Omega}\left[\frac{g_{1}\left(u_{1}^{*}, v_{1}^{*}\right)}{g_{1}\left(u, v_{1}^{*}\right)} g_{k}\left(u(x, t), v_{k}(x, t)\right)-\frac{c_{k} \delta_{k}}{p_{k}} v_{k}(x, t)\right] \mathrm{d} x \\
& +\int_{\Omega}\left(1-\frac{g_{1}\left(u_{1}^{*}, v_{1}^{*}\right)}{g_{1}\left(u, v_{1}^{*}\right)}\right) \nabla \cdot(D(u) \nabla u) \mathrm{d} x+\int_{\Omega}\left(1-\frac{w_{1}^{*}}{w_{1}}\right) \nabla \cdot\left(D\left(w_{1}\right) \nabla w_{1}\right) \mathrm{d} x \\
& +\frac{\delta_{1}}{p_{1}} \int_{\Omega}\left(1-\frac{w_{1}^{*}}{w_{1}}\right) \nabla \cdot\left(D\left(v_{1}\right) \nabla v_{1}\right) \mathrm{d} x+\sum_{k=2}^{n} \int_{\Omega} \nabla \cdot\left(D\left(w_{k}\right) \nabla w_{k}\right) \mathrm{d} x+\sum_{k=2}^{n} \frac{\delta_{k}}{p_{k}} \int_{\Omega} \nabla \cdot\left(D\left(v_{k}\right) \nabla v_{k}\right) \mathrm{d} x \\
& =\int_{\Omega}\left\{d u_{1}^{*}\left(1-\frac{u}{u_{1}^{*}}\right)\left(1-\frac{g_{1}\left(u_{1}^{*}, v_{1}^{*}\right)}{g_{1}\left(u, v_{1}^{*}\right)}\right)+g_{1}\left(u_{1}^{*}, v_{1}^{*}\right)\left[H\left(\frac{g_{1}\left(u(x, t), v_{1}(x, t)\right)}{g_{1}\left(u(x, t), v_{1}^{*}\right)}\right)-H\left(\frac{g_{1}\left(u_{1}^{*}, v_{1}^{*}\right)}{g_{1}\left(u(x, t), v_{1}^{*}\right)}\right)\right.\right. \\
& -H\left(\frac{g_{1}\left(u(x, t), v_{1}(x, t)\right)}{g_{1}\left(u_{1}^{*}, v_{1}^{*}\right)}\right)+H\left(\frac{g_{1}\left(u(x, t), v_{1}(x, t)\right)}{g_{1}\left(u_{1}^{*}, v_{1}^{*}\right)}\right)-H\left(\frac{g_{1}\left(u(x, t), v_{1}(x, t)\right) w_{1}^{*}}{g_{1}\left(u_{1}^{*}, v_{1}^{*}\right) w(x, t)}\right)-H\left(\frac{w_{1}(x, t)}{w_{1}^{*}}\right) \\
& \left.\left.+H\left(\frac{w_{1}(x, t)}{w_{1}^{*}}\right)-H\left(\frac{w_{1}(x, t) v_{1}^{*}}{v_{1}(x, t) w_{1}^{*}}\right)-H\left(\frac{v_{1}(x, t)}{v_{1}^{*}}\right)\right]\right\} \mathrm{d} x+\sum_{k=2}^{n} \int_{\Omega}\left[\frac{g_{1}\left(u_{1}^{*}, v_{1}^{*}\right)}{g_{1}\left(u, v_{1}^{*}\right)} g_{k}\left(u(x, t), v_{k}(x, t)\right)-\frac{c_{k} \delta_{k}}{p_{k}} v_{k}(x, t)\right] \mathrm{d} x \\
& +\int_{\Omega}\left(1-\frac{g_{1}\left(u_{1}^{*}, v_{1}^{*}\right)}{g_{1}\left(u, v_{1}^{*}\right)}\right) \nabla \cdot(D(u) \nabla u) \mathrm{d} x+\int_{\Omega}\left(1-\frac{w_{1}^{*}}{w_{1}}\right) \nabla \cdot\left(D\left(w_{1}\right) \nabla w\right) \mathrm{d} x+\frac{\delta_{1}}{p_{1}} \int_{\Omega}\left(1-\frac{v_{1}^{*}}{v_{1}}\right) \nabla \cdot\left(D\left(v_{1}\right) \nabla v_{1}\right) \mathrm{d} x \\
& +\sum_{k=2}^{n} \int_{\Omega} \nabla \cdot\left(D\left(w_{k}\right) \nabla w_{k}\right) \mathrm{d} x+\sum_{k=2}^{n} \frac{\delta_{k}}{p_{k}} \int_{\Omega} \nabla \cdot\left(D\left(v_{k}\right) \nabla v_{k}\right) \mathrm{d} x \\
& =\int_{\Omega}\left\{d u_{1}^{*}\left(1-\frac{u}{u_{1}^{*}}\right)\left(1-\frac{g_{1}\left(u_{1}^{*}, v_{1}^{*}\right)}{g_{1}\left(u, v_{1}^{*}\right)}\right)+g_{1}\left(u_{1}^{*}, v_{1}^{*}\right)\left[H\left(\frac{g_{1}\left(u(x, t), v_{1}(x, t)\right)}{g_{1}\left(u(x, t), v_{1}^{*}\right)}\right),\right.\right.
\end{aligned}
$$


From the hypothesis $\left(H_{1}\right)$, we can know the function $g_{1}\left(u, v_{1}^{*}\right)$ is strictly increasing in $u$. Hence, we have

$$
\left(1-\frac{u}{u_{1}^{*}}\right)\left(1-\frac{g_{1}\left(u_{1}^{*}, v_{1}^{*}\right)}{g_{1}\left(u, v_{1}^{*}\right)}\right) \leq 0,
$$

with equality only if $u=u_{1}^{*}$. Based on the assumption $\left(H_{3}\right)$, we can conclude that $g_{1}\left(u(x, t), v_{1}(x, t)\right) / g_{1}\left(u(x, t), v_{1}^{*}\right)$ lies between 1 and $\left(v_{1}(x, t) / v_{1}^{*}\right)$. Thus, by the fact that $H(z)$ is a monotone on each side of 1 , we can obtain that $H\left(g_{1}\left(u(x, t), v_{1}(x, t)\right) / g_{1}\left(u(x, t), v_{1}^{*}\right)\right) \leq H\left(v_{1}(x, t) / v_{1}^{*}\right)$. Meanwhile, for $k=2, \ldots, n$, we have

$$
\begin{aligned}
\frac{g_{1}\left(u_{1}^{*}, v_{1}^{*}\right)}{g_{1}\left(u, v_{1}^{*}\right)} g_{k}\left(u(x, t), v_{k}(x, t)\right)-\frac{c_{k} \delta_{k}}{p_{k}} v_{k}(x, t) & =\frac{c_{k} \delta_{k} g_{1}\left(u_{1}^{*}, v_{1}^{*}\right)}{p_{k} g_{1}\left(u, v_{1}^{*}\right)} v_{k}\left(\frac{p_{k}}{c_{k} \delta_{k}} \frac{g_{k}\left(u, v_{k}\right)}{v_{k}}-\frac{g_{1}\left(u, v_{1}^{*}\right)}{g_{1}\left(u_{1}^{*}, v_{1}^{*}\right)}\right) \\
& =\frac{c_{k} \delta_{k} g_{1}\left(u_{1}^{*}, v_{1}^{*}\right)}{p_{k} g_{1}\left(u, v_{1}^{*}\right)} v_{k}\left(\frac{g_{k}\left(u, v_{k}\right)}{v_{k}\left(\partial g_{k}(\lambda / d, 0) / \partial v_{k}\right)} \mathscr{R}_{k}-\frac{g_{1}\left(u, v_{1}^{*}\right)}{v_{1}^{*}\left(\partial g_{1}(\lambda / d, 0) / \partial v_{1}\right)} \mathscr{R}_{1}\right) \\
& =\frac{c_{k} \delta_{k} g_{1}\left(u_{1}^{*}, v_{1}^{*}\right)}{p_{k} g_{1}\left(u, v_{1}^{*}\right)} v_{k}\left(\alpha_{k} \mathscr{R}_{k}-\alpha_{1} \mathscr{R}_{1}\right),
\end{aligned}
$$

where $\alpha_{k}=\left(g_{k}\left(u, v_{k}\right) / v_{k}\left(\partial g_{k}(\lambda / d, 0)\right) / \partial v_{k}\right)$ and $\alpha_{1}=\left(g_{1}(u\right.$,

Under the condition $\left(H_{1}\right)$ and $\left(H_{4}\right)$, we have

$\left.\left.v_{1}^{*}\right) / v_{1}^{*}\left(\partial g_{1}(\lambda / d, 0)\right) / \partial v_{1}\right)$.

$$
\begin{aligned}
\frac{\alpha_{1}}{\alpha_{k}} & =\frac{\left(g_{1}\left(u, v_{1}^{*}\right)\right) / v_{1}^{*}}{\left(g_{k}\left(u, v_{k}\right)\right) / v_{k}} \frac{\partial g_{k}(\lambda / d, 0) / \partial v_{k}}{\partial g_{1}(\lambda / d, 0) / \partial v_{1}} \geq \frac{\partial g_{1}\left(u, v_{1}^{*}\right) / \partial v_{1}}{g_{k}\left(u, v_{k}\right) / v_{k}} \frac{\partial g_{k}(\lambda / d, 0) / \partial v_{k}}{\partial g_{1}(\lambda / d, 0) / \partial v_{1}} \\
& \geq \frac{\partial g_{1}\left(u, v_{1}^{*}\right) / \partial v_{1}}{\eta_{k}(\lambda / d)} \frac{\partial g_{k}(\lambda / d, 0) / \partial v_{k}}{\partial g_{1}(\lambda / d, 0) / \partial v_{1}} \\
& \geq \frac{p_{k}}{c_{k} \delta_{k}} \frac{c_{1} \delta_{1}}{p_{1}} \frac{\partial g_{k}(\lambda / d, 0) / \partial v_{k}}{\partial g_{1}(\lambda / d, 0) / \partial v_{1}}=\frac{\mathscr{R}_{k}}{\mathscr{R}_{1}},
\end{aligned}
$$

which yields that

$$
\sum_{k=2}^{n} \int_{\Omega}\left[\frac{g_{1}\left(u_{1}^{*}, v_{1}^{*}\right)}{g_{1}\left(u, v_{1}^{*}\right)} g_{k}\left(u(x, t), v_{k}(x, t)\right)-\frac{c_{k} \delta_{k}}{p_{k}} v_{k}(x, t)\right] \mathrm{d} x \leq 0 .
$$

Therefore, we obtain

$$
\begin{aligned}
\frac{\mathrm{d} V(t)}{\mathrm{d} t} \leq & -g_{1}\left(u_{1}^{*}, v_{1}^{*}\right) \int_{\Omega}\left[H\left(\frac{g_{1}\left(u^{*}, v_{1}^{*}\right)}{g_{1}\left(u, v_{1}^{*}\right)}\right)+H\left(\frac{w_{1}(x, t) v_{1}^{*}}{v_{1}(x, t) w_{1}^{*}}\right)+H\left(\frac{g_{1}\left(u(x, t), v_{1}(x, t)\right) w_{1}^{*}}{g_{1}\left(u_{1}^{*}, v_{1}^{*}\right) w_{1}(x, t)}\right)\right] \mathrm{d} x \\
& +\int_{\Omega}\left(1-\frac{g_{1}\left(u_{1}^{*}, v_{1}^{*}\right)}{g_{1}\left(u, v_{1}^{*}\right)}\right) \nabla \cdot(D(u) \nabla u) \mathrm{d} x+\int_{\Omega}\left(1-\frac{w_{1}^{*}}{w_{1}}\right) \nabla \cdot\left(D\left(w_{1}\right) \nabla w\right) \mathrm{d} x \\
& +\frac{\delta_{1}}{p_{1}} \int_{\Omega}\left(1-\frac{v_{1}^{*}}{v_{1}}\right) \nabla \cdot\left(D\left(v_{1}\right) \nabla v_{1}\right) \mathrm{d} x+\sum_{k=2}^{n} \int_{\Omega} \nabla \cdot\left(D\left(w_{k}\right) \nabla w_{k}\right) \mathrm{d} x+\sum_{k=2}^{n} \frac{\delta_{k}}{p_{k}} \int_{\Omega} \nabla \cdot\left(D\left(v_{k}\right) \nabla v_{k}\right) \mathrm{d} x .
\end{aligned}
$$

It follows from Lemma 2 and Remark 1 that

$$
\frac{\mathrm{d} V(t)}{\mathrm{d} t} \leq 0 \text {. }
$$

Hence, $E_{1}$ is stable, and $((\mathrm{d} V(t)) / \mathrm{d} t)=0$ iff $u=u_{1}^{*}$, $w=w_{1}^{*}, v=v_{1}^{*}$, and $w_{k}=0=v_{k}$ for $k=2, \ldots, n$. It is easy to confirm that the singleton $\left\{E_{1}\right\}$ is just the largest compact invariant set in $\{(u, w, v) \mid((\mathrm{d} V(t)) / \mathrm{d} t)=0\}$. When $\mathscr{R}_{1}>1$ and $\mathscr{R}_{1}>\mathscr{R}_{k}$ hold for $k=2, \ldots, n$, we can know that the infection steady state $E_{1}$ of model (7) is globally asymptotically stable by the LaSalle's invariance principle. The proof of Theorem 6 is complete.

Theorem 7. Assume $\mathscr{R}_{i}<1$ for $i=1, \ldots, n$. Then, the steady state $E_{0}^{n}$ of model (7) is globally asymptotically stable. 
Proof. Define

$$
V(t)=\int_{\Omega} \sum_{i=1}^{n}\left[w_{i}(x, t)+\frac{\delta_{i}}{p_{i}} v_{i}(x, t)\right] \mathrm{d} x,
$$

which is called the Lyapunov functional. The rest of the proof is similar to Theorem 4. So, we omit it. This completes the proof of Theorem 7 .

Remark 2. From Theorem 7, when the basic reproductive number of each strain is less than 1 , we can see that all viral strains die out.

\section{Numerical Calculations and Simulations}

In this section, we perform some numerical simulations that illustrate and supplement the analytic results given in the previous sections. In what follows, we perform the numerical simulations from the two aspects of global threshold dynamics and competitive exclusion, respectively.

Firstly, we carry out the numerical simulation for global threshold dynamics. Taking the incidence function as $g(u(x, t), \quad v(x, t))=(\beta u(x, t) v(x, t)) /(1+\quad a u(x, t)+b v$ $(x, t)+a b u(x, t) v(x, t))$, we can change model (1) into the following form:

$$
\left\{\begin{array}{l}
\frac{\partial u(x, t)}{\partial t}=\nabla \cdot\left(D_{u}(u) \nabla u\right)+\lambda-d u(x, t)-\frac{\beta u(x, t) v(x, t)}{1+a u(x, t)+b v(x, t)+a b u(x, t) v(x, t)} \\
\frac{\partial w(x, t)}{\partial t}=\nabla \cdot\left(D_{w}(w) \nabla w\right)+\frac{\beta u(x, t) v(x, t)}{1+a u(x, t)+b v(x, t)+a b u(x, t) v(x, t)}-\delta w(x, t), \\
\frac{\partial v(x, t)}{\partial t}=\nabla \cdot\left(D_{v}(v) \nabla v\right)+p w(x, t)-c v(x, t),
\end{array}\right.
$$

with Neumann boundary conditions (2) and initial conditions (3). From (10), together with direct computation, we get the basic reproduction number $\mathscr{R}_{0}=(p \lambda \beta /(c \delta(d+a \lambda)))$ for system (59). Meanwhile, the equilibria are given out by $E_{0}=((\lambda / d), 0,0)$ and $E^{*}=\left(u^{*}, w^{*}, v^{*}\right)$, where $w^{*}=((\lambda-$ $\left.\left.d u^{*}\right) / \delta\right), v^{*}=\left(p\left(\lambda-d u^{*}\right) / c \delta\right)$, and

$$
u^{*}=\frac{p a b \lambda+a c \delta-p \beta-p b d+\sqrt{(p a b \lambda+a c \delta-p \beta-p b d)^{2}+4 p a b d(p b \lambda+c \delta)}}{2 p a b d}
$$

is a unique positive solution of the following quadratic equation:

$$
p a b d u^{2}+(p \beta+p b d-p a b \lambda-a c \delta) u-p b \lambda-c \delta=0 .
$$

It is not hard to verify that if $\mathscr{R}_{0}>1$, then $\lambda-d u^{*}>0$. So, $E^{*}=\left(u^{*}, w^{*}, v^{*}\right)$ if $\mathscr{R}_{0}>1$ is a unique interior equilibrium of system (59).

For simplicity of the numerical illustration, we consider only one spatial dimension with spatial domain $x \in \Omega=[0,6]$. The numerical simulations are observed for a time duration of 500 or 1000 days. Here, we have to point out that some parameter values are taken from the literature [24]. Then, we set choose the parameter values as $m_{1}=m_{2}=m_{3}=2, \lambda=10, d=0.09, \beta=0.0005, \delta=0.4$, $c=2.4, \quad p=300, a=0.5$, and $b=0.4$. Through direct computation, we obtain $\mathscr{R}_{0}=0.3070<1$ and $E_{0}=(111.1111,0,0)$. From Theorem 4 , we can know that infection-free equilibrium $E_{0}$ of model (59) is globally asymptotically stable, see Figure 1. Further, we choose the parameter values as $m_{1}=m_{2}=m_{3}=2, \lambda=10, d=0.09$, $\beta=0.0025, \quad \delta=0.4, \quad c=2.4, \quad p=300, \quad a=0.0015$, and $b=0.4$. Through direct computation, we obtain $\mathscr{R}_{0}=74.4048>1$ and $E^{*}=(104.9054,1.3963,174.5354)$. From Theorem 5, we can know that the infection equilibrium $E^{*}$ of model (59) is globally asymptotically stable, see Figure 2.

Secondly, we perform the numerical simulation for competitive exclusion. Taking the incidence function as $g_{i}(u(x, t), v(x, t))=\left(\beta_{i} u(x, t) v_{i}(x, t)\right) /\left(1+a_{i} u(x, t)+\right.$ $\left.b_{i} v_{i}(x, t)+a_{i} b_{i} u(x, t) v_{i}(x, t)\right)$ and $n=3$, we can change model (7) into the following form: 

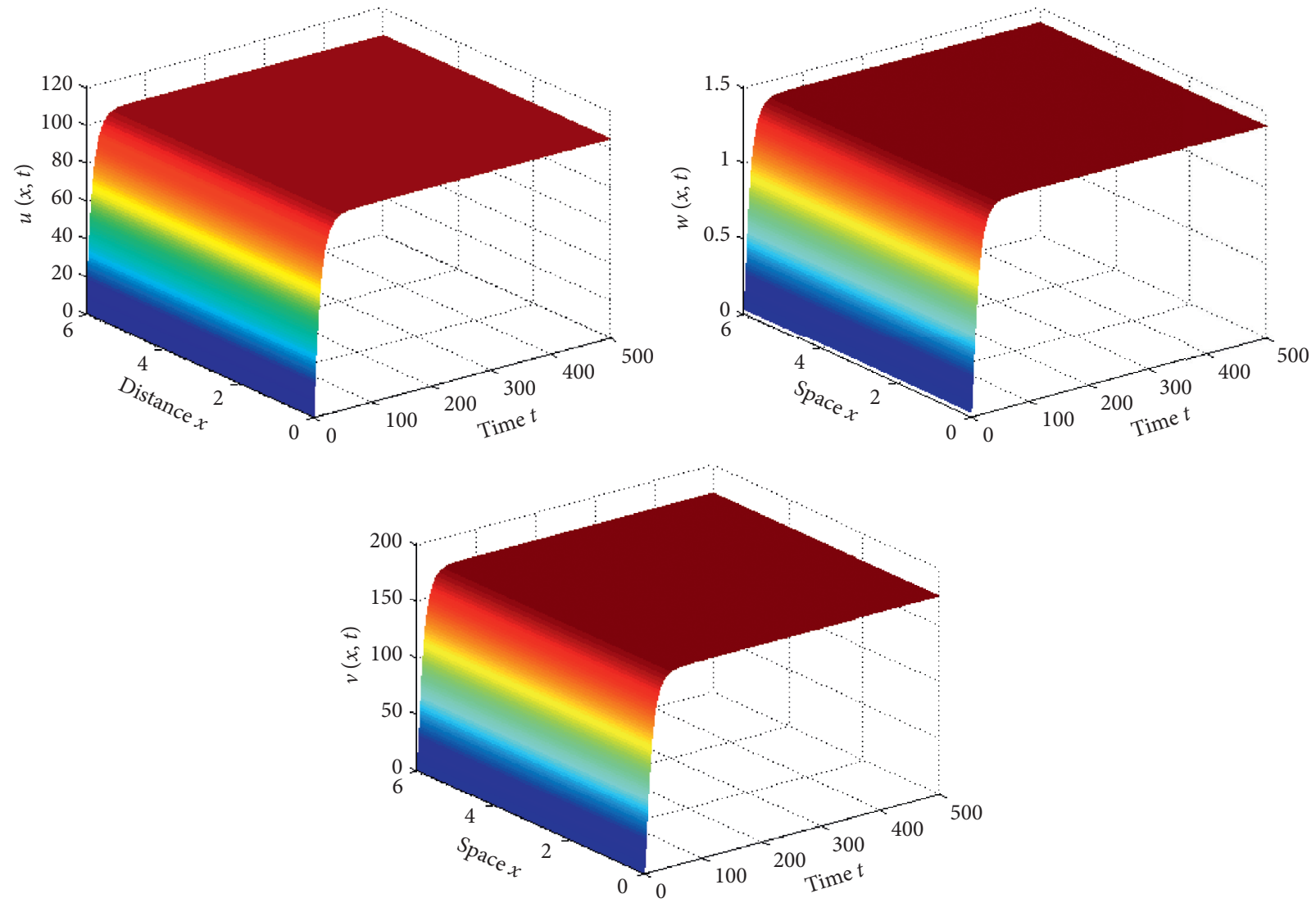

Figure 2: Taking $m_{1}=m_{2}=m_{3}=2, \lambda=10, d=0.09, \beta=0.0025, \delta=0.4, c=2.4, p=300, a=0.0015$, and $b=0.4$, we have $\mathscr{R}_{0}=$ $74.4048>1$ and the infection equilibrium $E^{*}=(104.9054,1.3963,174.5354)$ of model (59) is globally asymptotically stable.

$$
\left\{\begin{array}{l}
\frac{\partial u(x, t)}{\partial t}=\nabla \cdot\left(D_{u}(u) \nabla u\right)+\lambda-d u(x, t)-\sum_{i=1}^{3} \frac{\beta_{i} u(x, t) v_{i}(x, t)}{1+a_{i} u(x, t)+b_{i} v_{i}(x, t)+a_{i} b_{i} u(x, t) v_{i}(x, t)}, \\
\frac{\partial w_{i}(x, t)}{\partial t}=\nabla \cdot\left(D_{w_{i}}\left(w_{i}\right) \nabla w_{i}\right)+\frac{\beta_{i} u(x, t) v_{i}(x, t)}{1+a_{i} u(x, t)+b_{i} v_{i}(x, t)+a_{i} b_{i} u(x, t) v_{i}(x, t)}-\delta_{i} w_{i}(x, t), \\
\frac{\partial v_{i}(x, t)}{\partial t}=\nabla \cdot\left(D_{v_{i}}\left(v_{i}\right) \nabla v_{i}\right)+p_{i} w_{i}(x, t)-c_{i} v_{i}(x, t), \quad i=1,2,3,
\end{array}\right.
$$

with Neumann boundary conditions (40) and initial conditions (41). By $\mathscr{R}_{i}=\left(p_{i} / c_{i} \delta_{i}\right)\left(\partial g_{i}(\lambda / d, 0) / \partial v_{i}\right)$, together with direct computation, we can get the basic reproduction number for viral strain $i$ for model (62) as $\mathscr{R}_{i}=\left(p_{i} \lambda \beta_{i}\right) /\left(c_{i} \delta_{i}\left(d+a_{i} \lambda\right)\right)$. Meanwhile, we can obtain the equilibria $E_{0}^{3}=((\lambda / d), \mathbf{0 , 0})$, where $\mathbf{0}$ is a 3-dimensional vector of zeros, and $E_{i}=\left(u^{*}, 0, w_{i}^{*}, 0,0, v_{i}^{*}, 0\right)$ for each strain $i(i=1,2,3)$, where $w_{i}^{*}=\left(\left(\lambda-d u^{*}\right) / \delta_{i}\right), v_{i}^{*}=\left(\left(p_{i}\right.\right.$ $\left.\left.\left(\lambda-d u^{*}\right)\right) / c_{i} \delta_{i}\right)$, and

$$
u^{*}=\frac{\lambda p_{i} a_{i} b_{i}+a_{i} c_{i} \delta_{i}-p_{i} \beta_{i}-d p_{i} b_{i}+\sqrt{\left(\lambda p_{i} a_{i} b_{i}+a_{i} c_{i} \delta_{i}-p_{i} \beta_{i}-d p_{i} b_{i}\right)^{2}+4 d \lambda p_{i} a_{i} b_{i}\left(p_{i} b_{i}+c_{i} \delta_{i}\right)}}{2 d p_{i} a_{i} b_{i}} .
$$

Choosing the parameter values as $m_{1}=m_{2}=m_{3}=2$, $\lambda=10$, and $d=0.09$ and $\beta_{1}=0.0025, \delta_{1}=0.4, c_{1}=2.4$, $p_{1}=300, a_{1}=0.0015$, and $b_{1}=0.4$ for strain $1 ; \beta_{2}=0.0015$, $\delta_{2}=0.6, c_{2}=2.4, p_{2}=250, a_{2}=0.5$, and $b_{2}=0.2$ for strain $2 ; \beta_{3}=0.0002, \delta_{3}=0.2, c_{3}=3.2, p_{3}=320, a_{3}=0.8$, and $b_{3}=0.6$ for strain 3 , we can obtain $\mathscr{R}_{1}=74.4048>1$, 

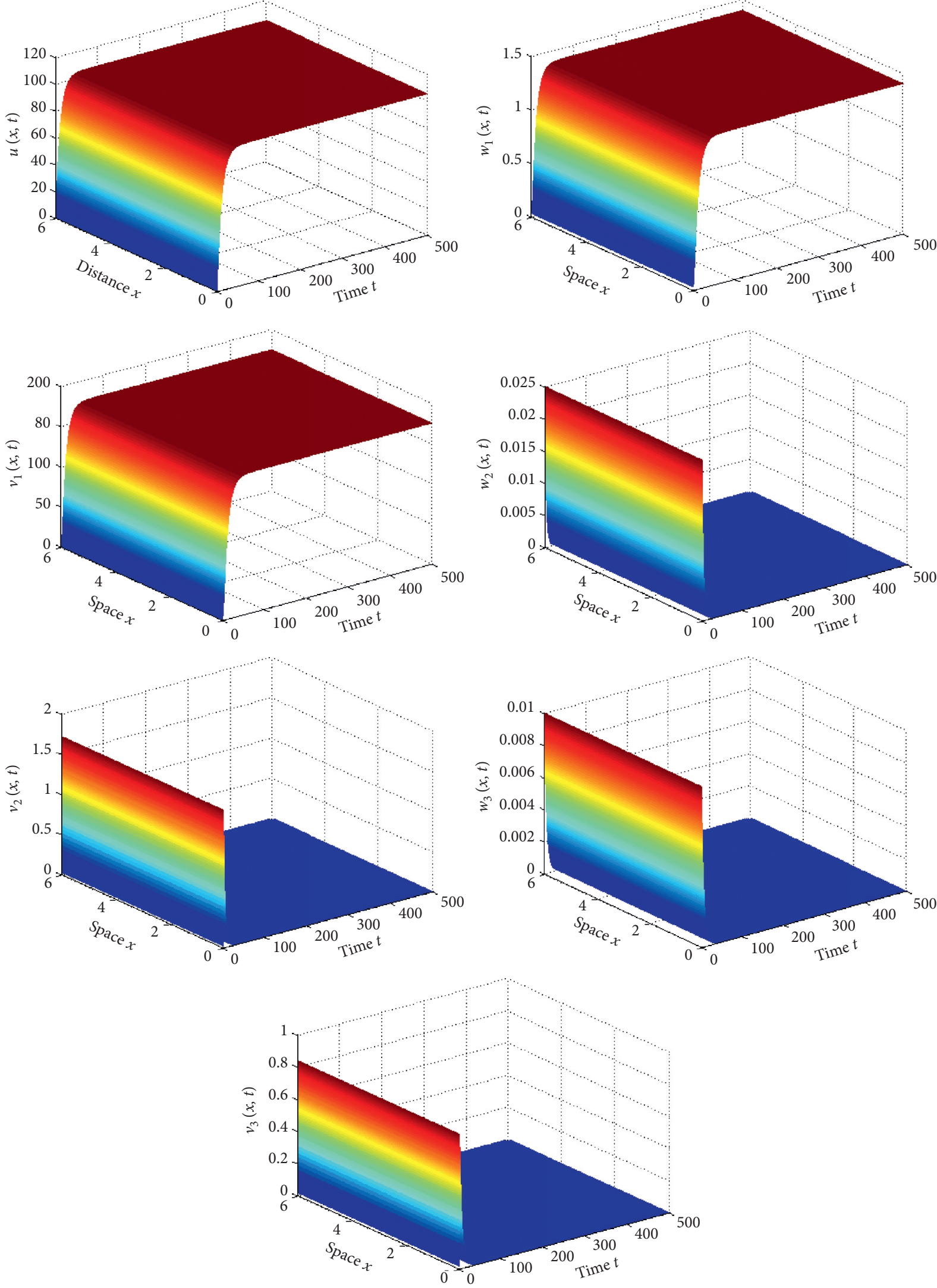

Figure 3: Taking $m_{1}=m_{2}=m_{3}=2, \lambda=10$, and $d=0.09$ and $\beta_{1}=0.0025, \delta_{1}=0.4, c_{1}=2.4, p_{1}=300, a_{1}=0.0015$, and $b_{1}=0.4$ for strain $1 ; \beta_{2}=0.0015, \delta_{2}=0.6, c_{2}=2.4, p_{2}=250, a_{2}=0.5$, and $b_{2}=0.2$ for strain $2 ; \beta_{3}=0.0002, \delta_{3}=0.2, c_{3}=3.2, p_{3}=320, a_{3}=0.8$, and $b_{3}=0.6$ for strain 3 , we have $\mathscr{R}_{0}=74.4048>1, \quad \mathscr{R}_{2}=0.5147<1, \quad \mathscr{R}_{3}=0.1357<1$, and the infection equilibrium $E^{1}=(104.9054,1.3963,0,0,174.5354,0,0)$ of model $(62)$ is globally asymptotically stable. 

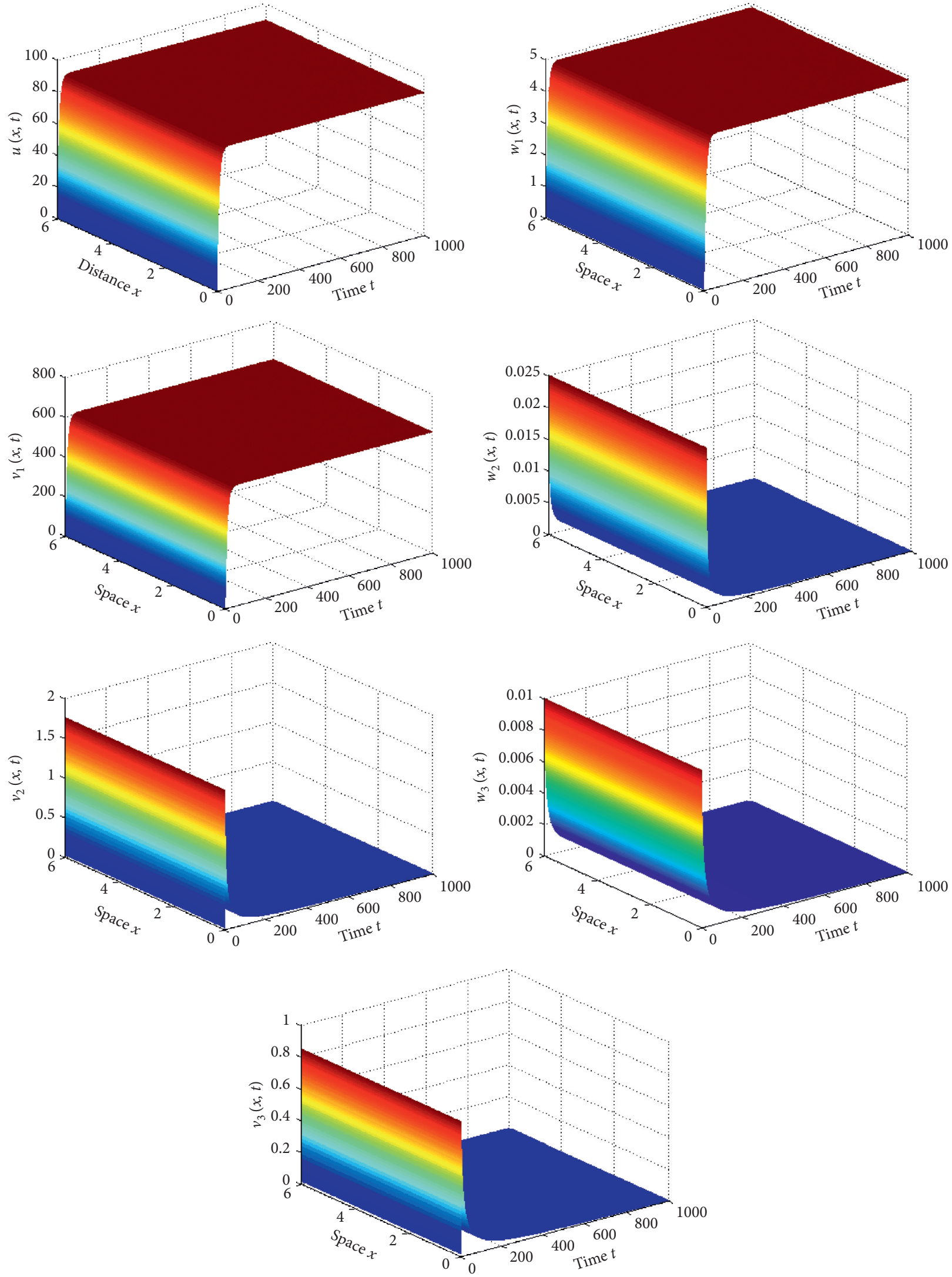

Figure 4: Taking $m_{1}=m_{2}=m_{3}=2, \lambda=10$, and $d=0.09$ and $\beta_{1}=0.0025, \delta_{1}=0.4, c_{1}=2.4, p_{1}=300, a_{1}=0.0015$, and $b_{1}=0.1$ for strain $1 ; \beta_{2}=0.0015, \delta_{2}=0.6, c_{2}=2.4, p_{2}=250, a_{2}=0.25$, and $b_{2}=0.2$ for strain $2 ; \beta_{3}=0.0002, \delta_{3}=0.2, c_{3}=3.2, p_{3}=320, a_{3}=0.092$, and $b_{3}=0.6$ for strain 3 , we have $\mathscr{R}_{0}=74.4048>1, \mathscr{R}_{2}=1.0055>1, \quad \mathscr{R}_{3}=1.0204>1$, and the infection equilibrium $E^{1}=(89.5396,4.8536,0,0,606.6994,0,0)$ of model (62) is globally asymptotically stable. 

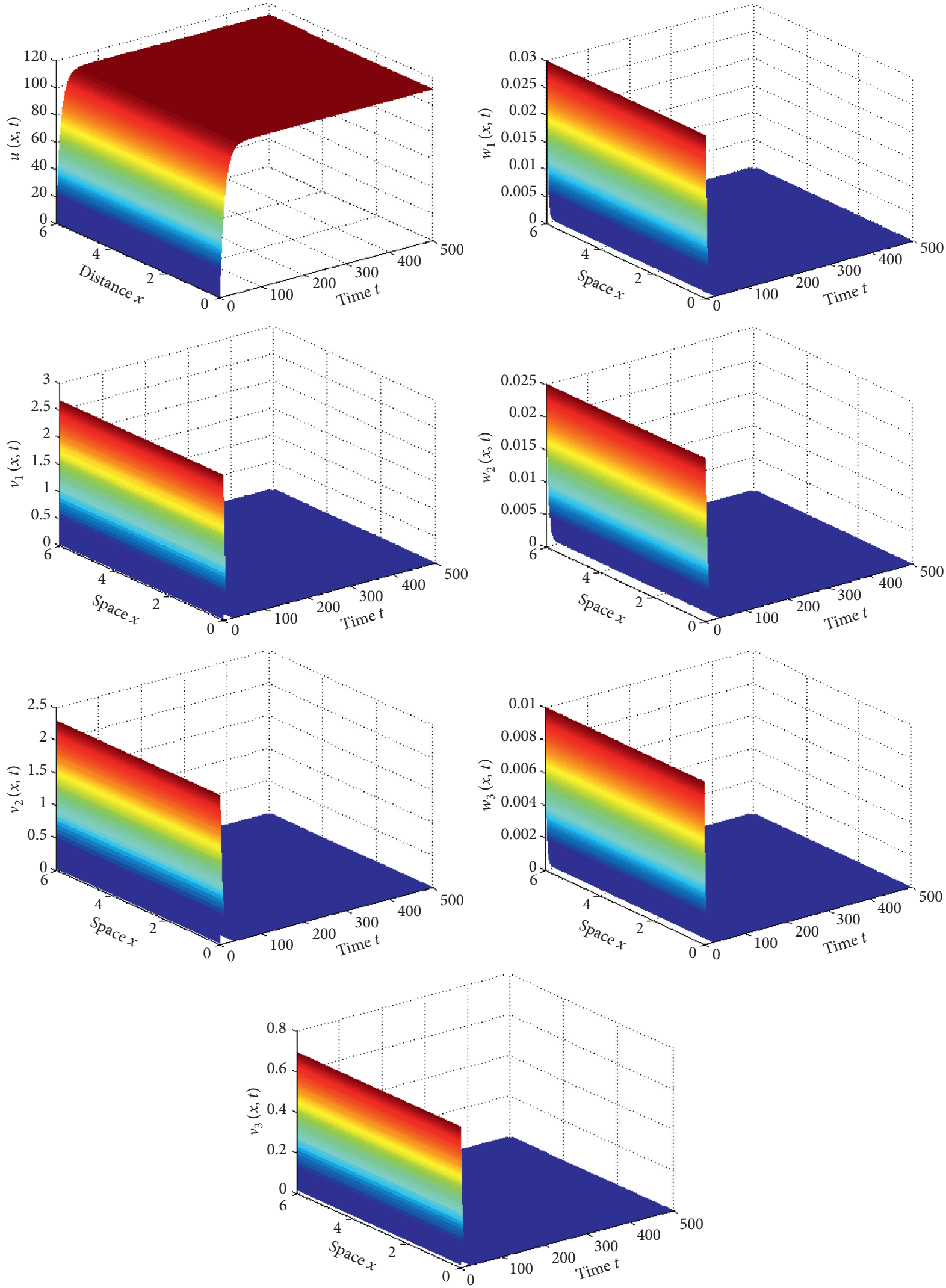

Figure 5: Taking $m_{1}=m_{2}=m_{3}=2, \lambda=10$, and $d=0.09$ and $\beta_{1}=0.0005, \delta_{1}=0.4, c_{1}=2.4, p_{1}=300, a_{1}=0.5$, and $b_{1}=0.4$ for strain 1 ; $\beta_{2}=0.0015, \delta_{2}=0.6, c_{2}=2.4, p_{2}=250, a_{2}=0.5$, and $b_{2}=0.2$ for strain $2 ; \beta_{3}=0.0002, \delta_{3}=0.2, c_{3}=3.2, p_{3}=320, a_{3}=0.8$, and $b_{3}=0.6$ for strain 3, we have $\mathscr{R}_{1}=0.3070<1, \mathscr{R}_{2}=0.5147<1, \mathscr{R}_{3}=0.1357<1$, and the infection-free equilibrium $E_{0}^{3}=(111.1111,0,0,0,0,0,0)$ of model (62) is globally asymptotically stable. 
$\mathscr{R}_{2}=0.5147<1, \quad \mathscr{R}_{3}=0.1357<1, \quad$ and $E^{1}=(104.9054$, $1.3963,0,0,174.5354,0,0)$ by direct computation. Moreover, we can get that

$$
\begin{aligned}
\frac{\alpha_{1}}{\alpha_{k}}= & \frac{1+a_{i} u(x, t)+b_{i} v_{i}(x, t)+a_{i} b_{i} u(x, t) v_{i}(x, t)}{1+a_{1} u(x, t)+b_{1} v_{1}^{*}+a_{1} b_{1} u(x, t) v_{1}^{*}} \\
& \geq \frac{1}{1+a_{1}(\lambda / d)+b_{1} v_{1}^{*}+a_{1} b_{1}(\lambda / d) v_{1}^{*}}=0.0121, \quad k=2,3,
\end{aligned}
$$

which yields that $\left(\alpha_{1} / \alpha_{2}\right) \times \mathscr{R}_{1}=0.9003>0.5147=\mathscr{R}_{2}$ and $\left(\alpha_{1} / \alpha_{3}\right) \times \mathscr{R}_{1}=0.9003>0.1357=\mathscr{R}_{3}$. From Theorem 6, we can know that the infection equilibrium $E^{1}$ of model (62) is globally asymptotically stable, which implies that strain one persists, while strains 2 and 3 die out. These facts are numerically confirmed in Figure 3.

Further, we take another parameter value as $\beta_{1}=0.0025$, $\delta_{1}=0.4, c_{1}=2.4, p_{1}=300, a_{1}=0.0015$, and $b_{1}=0.1$ for strain $1 ; \beta_{2}=0.0015, \delta_{2}=0.6, c_{2}=2.4, p_{2}=250, a_{2}=0.25$, and $b_{2}=0.2$ for strain $2 ; \beta_{3}=0.0002, \delta_{3}=0.2, c_{3}=3.2$, $p_{3}=320, a_{3}=0.092$, and $b_{3}=0.6$ for strain 3. By direct computation again, we can also obtain $\mathscr{R}_{1}=74.4048>1$, $\mathscr{R}_{2}=1.0055>1, \mathscr{R}_{3}=1.0204>1, E^{1}=(89.5396,4.8536$, $0,0,606.6994,0,0)$, and

$$
\begin{aligned}
\frac{\alpha_{1}}{\alpha_{k}}= & \frac{1+a_{i} u(x, t)+b_{i} v_{i}(x, t)+a_{i} b_{i} u(x, t) v_{i}(x, t)}{1+a_{1} u(x, t)+b_{1} v_{1}^{*}+a_{1} b_{1} u(x, t) v_{1}^{*}} \\
& \geq \frac{1}{1+a_{1}(\lambda / d)+b_{1} v_{1}^{*}+a_{1} b_{1}(\lambda / d) v_{1}^{*}}=0.0139, \quad k=2,3,
\end{aligned}
$$

which yields that $\left(\alpha_{1} / \alpha_{2}\right) \times \mathscr{R}_{1}=1.0342>1.0055=\mathscr{R}_{2}$ and $\left(\alpha_{1} / \alpha_{3}\right) \times \mathscr{R}_{1}=1.0342>1.0204=\mathscr{R}_{3}$. From Theorem 6, we can know that the infection equilibrium $E^{1}$ of model (62) is globally asymptotically stable, which implies that strain one persists, while strains 2 and 3 die out. These facts are numerically confirmed in Figure 4.

Next, we take the parameter values as $m_{1}=m_{2}=m_{3}=2$, $\lambda=10$, and $d=0.09$ and $\beta_{1}=0.0005, \delta_{1}=0.4, c_{1}=2.4$, $p_{1}=300, a_{1}=0.5$, and $b_{1}=0.4$ for strain $1 ; \beta_{2}=0.0015$, $\delta_{2}=0.6, c_{2}=2.4, p_{2}=250, a_{2}=0.5$, and $b_{2}=0.2$ for strain 2; $\beta_{3}=0.0002, \delta_{3}=0.2, c_{3}=3.2, p_{3}=320, a_{3}=0.8$, and $b_{3}=0.6$ for strain 3 . By direct computation, we can get $\mathscr{R}_{1}=0.3070<1, \mathscr{R}_{2}=0.5147<1, \mathscr{R}_{3}=0.1357<1$, and $E_{0}^{3}=(111.1111,0,0,0,0,0,0)$. From Theorem 7 , we can know that infection-free equilibrium $E_{0}^{3}$ of model (62) is globally asymptotically stable, which implies that all viral strains die out. These facts are numerically confirmed in Figure 5.

\section{Conclusion}

In this paper, we investigate the global threshold dynamics in single-strain viral infection models (1) and competitive exclusion in multistrain viral infection models (7) under the homogeneous Neumann boundary conditions.

For model (1), by constructing a Lyapunov functional, we obtain that the infection-free equilibrium $E_{0}$ of model (1) is globally asymptotically stable if the basic reproduction number $\mathscr{R}_{0}<1$, and the infection equilibrium $E^{*}$ of model (1) is globally asymptotically stable if the basic reproduction number $\mathscr{R}_{0}>1$. These facts are numerically confirmed in Figures 1 and 2, respectively. For model (7), by constructing a Lyapunov functional, we also obtain that the steady state $E_{1}$ of model (7) is globally asymptotically stable if $\mathscr{R}_{1}>1$ and $\mathscr{R}_{1}>\mathscr{R}_{k}$ for $k=2, \ldots, n$, which implies that strain one persists, while strains $k=2, \ldots, n$ die out. These facts are numerically confirmed in Figures 3 and 4 . Meanwhile, the steady state $E_{0}^{n}$ of model (7) is globally asymptotically stable if $\mathscr{R}_{i}<1$ for $i=1, \ldots, n$, which implies that all viral strains die out. These facts are numerically confirmed in Figure 5. Here, we have to point out that the incidence function in the present paper is general and performed the numerical simulation for global threshold dynamics and competitive exclusion. So, our results in the present paper are new and can also be applied to other reaction-diffusion systems. Of course, we hope that our work could be instructive to study the dynamics of the viral infection model.

\section{Data Availability}

The data used to support the findings of this study are included within the article.

\section{Conflicts of Interest}

The authors declare that they have no conflicts of interest.

\section{Acknowledgments}

The work was supported by the National Natural Science Foundation of China (no. 11761038), the Science and Technology Project of Department of Education of Jiangxi Province (no. GJJ180583), and Natural Science Foundation of Jiangxi Province of China (no. 20171BAB202010).

\section{References}

[1] M. A. Nowak and C. R. M. Bangham, "Population dynamics of immune responses to persistent viruses," Science, vol. 272, no. 5258 , pp. 74-79, 1996.

[2] M. A. Nowak, S. Bonhoeffer, A. M. Hill, R. Boehme, H. C. Thomas, and H. McDade, "Viral dynamics in hepatitis B virus infection," Proceedings of the National Academy of Sciences, vol. 93, no. 9, pp. 4398-4402, 1996.

[3] P. De Leenheer and H. L. Smith, "Virus dynamics: a global analysis," SIAM Journal on Applied Mathematics, vol. 63, no. 4, pp. 1313-1327, 2003.

[4] X. Song and A. U. Neumann, "Global stability and periodic solution of the viral dynamics," Journal of Mathematical Analysis and Applications, vol. 329, no. 1, pp. 281-297, 2007.

[5] K. Wang, W. Wang, and S. Song, "Dynamics of an HBV model with diffusion and delay," Journal of Theoretical Biology, vol. 253, no. 1, pp. 36-44, 2008.

[6] S. Wang, J. Zhang, F. Xu, and X. Song, "Dynamics of virus infection models with density-dependent diffusion," Computers \& Mathematics with Applications, vol. 74, no. 10, pp. 2403-2422, 2017.

[7] S. Wang, J. Zhang, F. Xu, and X. Song, "Dynamics of virus infection models with density-dependent diffusion and Beddington-DeAngelis functional response," Mathematical 
Methods in the Applied Sciences, vol. 40, no. 15, pp. 5593-5612, 2017.

[8] J. Wang, M. Guo, X. Liu, and Z. Zhao, "Threshold dynamics of HIV-1 virus model with cell-to-cell transmission, cell-mediated immune responses and distributed delay," Applied Mathematics and Computation, vol. 291, pp. 149-161, 2016.

[9] W. Wang and W. Ma, "A diffusive HIV infection model with nonlocal delayed transmission," Applied Mathematics Letters, vol. 75, pp. 96-101, 2018.

[10] Q. Hu, Z. Hu, and F. Liao, "Stability and Hopf bifurcation in a HIV-1 infection model with delays and logistic growth," Mathematics and Computers in Simulation, vol. 128, pp. 2641, 2016.

[11] Y. Wang, J. Liu, and J. M. Heffernan, "Viral dynamics of an HTLV-I infection model with intracellular delay and CTL immune response delay," Journal of Mathematical Analysis and Applications, vol. 459, no. 1, pp. 506-527, 2018.

[12] W. Wang, W. Ma, and X. Lai, "Repulsion effect on superinfecting virions by infected cells for virus infection dynamic model with absorption effect and chemotaxis," Nonlinear Analysis: Real World Applications, vol. 33, pp. 253-283, 2017.

[13] W. Wang and W. Ma, "Travelling wave solutions for a nonlocal dispersal HIV infection dynamical model," Journal of Mathematical Analysis and Applications, vol. 457, no. 1, pp. 868-889, 2018.

[14] W. Wang, Y. Cai, M. Wu, K. Wang, and Z. Li, "Complex dynamics of a reaction-diffusion epidemic model," Nonlinear Analysis: Real World Applications, vol. 13, no. 5, pp. 22402258, 2012.

[15] M. C. Lombardo, R. Barresi, E. Bilotta, F. Gargano, P. Pantano, and M. Sammartino, "Demyelination patterns in a mathematical model of multiple sclerosis," Journal of Mathematical Biology, vol. 75, no. 2, pp. 373-417, 2017.

[16] X. Tang and J. Li, "Chemotaxis induced Turing bifurcation in a partly diffusive bacterial and viral diseases propagation model," Applied Mathematics Letters, vol. 100, Article ID 106037, 2020.

[17] J. Murray, "Mathematical biology II: spatial models and biomedical applications," in Biomathematics, Springer, New York, NY, USA, 3rd edition, 2003.

[18] C. V. Pao and W. H. Ruan, "Quasilinear parabolic and elliptic systems with mixed quasimonotone functions," Journal of Differential Equations, vol. 255, no. 7, pp. 1515-1553, 2013.

[19] Y. Chen and Y. Zhu, "Dynamics of prey-predator n-species models with density dependent diffusion," Computers \& Mathematics with Applications, vol. 72, no. 6, pp. 1727-1742, 2016.

[20] C. V. Pao and W. H. Ruan, "Dynamics of degenerate quasilinear reaction diffusion systems with nonnegative initial functions," Journal of Differential Equations, vol. 263, no. 11, pp. 7709-7752, 2017.

[21] C. V. Pao, "Global attractors of some predator-prey reactiondiffusion systems with density-dependent diffusion and timedelays," Journal of Mathematical Analysis and Applications, vol. 464, no. 1, pp. 164-187, 2018.

[22] R. Xu, "Global stability of an HIV-1 infection model with saturation infection and intracellular delay," Journal of Mathematical Analysis and Applications, vol. 375, no. 1, pp. 75-81, 2011.

[23] X. Wang, Y. Tao, and X. Song, "Global stability of a virus dynamics model with Beddington-DeAngelis incidence rate and CTL immune response," Nonlinear Dynamics, vol. 66, no. 4, pp. 825-830, 2011.
[24] X. Zhou and J. Cui, "Global stability of the viral dynamics with Crowley-Martin functional response," Bulletin of the Korean Mathematical Society, vol. 48, no. 3, pp. 555-574, 2011.

[25] A. Korobeinikov, "Global asymptotic properties of virus dynamics models with dose-dependent parasite reproduction and virulence and non-linear incidence rate," Mathematical Medicine and Biology, vol. 26, no. 3, pp. 225-239, 2009.

[26] G. Huang, Y. Takeuchi, and W. Ma, "Lyapunov functionals for delay differential equations model of viral infections," SIAM Journal on Applied Mathematics, vol. 70, no. 7, pp. 2693-2708, 2010.

[27] C. C. McCluskey and Y. Yang, "Global stability of a diffusive virus dynamics model with general incidence function and time delay," Nonlinear Analysis: Real World Applications, vol. 25, pp. 64-78, 2015.

[28] K. Hattaf and N. Yousfi, "A generalized HBV model with diffusion and two delays," Computers \& Mathematics with Applications, vol. 69, no. 1, pp. 31-40, 2015.

[29] H. Miao, Z. Teng, X. Abdurahman, and Z. Li, "Global stability of a diffusive and delayed virus infection model with general incidence function and adaptive immune response," Computational and Applied Mathematics, vol. 37, no. 3, pp. 3780-3805, 2018.

[30] G. F. Gause, The Struggle for Existence, Williams \& Wilkins, Baltimore, MD, USA, 1936.

[31] H. J. Bremermann and H. R. Thieme, "A competitive exclusion principle for pathogen virulence," Journal of Mathematical Biology, vol. 27, no. 2, pp. 179-190, 1989.

[32] P. De Leenheer and S. S. Pilyugin, "Multistrain virus dynamics with mutations: a global analysis," Mathematical Medicine and Biology, vol. 25, no. 4, pp. 285-322, 2008.

[33] Z. Feng and J. X. Velasco-Hernández, "Competitive exclusion in a vector-host model for the dengue fever," Journal of Mathematical Biology, vol. 35, no. 5, pp. 523-544, 1997.

[34] A. Iggidr, J. C. Kamgang, G. Sallet, and J. J. Tewa, "Global analysis of new malaria intrahost models with a competitive exclusion principle," SIAM Journal on Applied Mathematics, vol. 67, no. 1, pp. 260-278, 2006.

[35] D. Bichara, A. Iggidr, and G. Sallet, "Global analysis of multistrains SIS, SIR and MSIR epidemic models," Journal of Applied Mathematics and Computing, vol. 44, no. 1-2, pp. 273-292, 2014.

[36] X.-C. Duan, J.-F. Yin, X.-Z. Li, and M. Martcheva, "Competitive exclusion in a multi-strain virus model with spatial diffusion and age of infection," Journal of Mathematical Analysis and Applications, vol. 459, no. 2, pp. 717-742, 2018.

[37] M. T. Meehana, D. G. Cocksb, and E. S. McBryde, "Global stability of the multi-strain Kermack-McKendrick (renewal) epidemic model," 2019, https://arxiv.org/abs/1710.06984. 